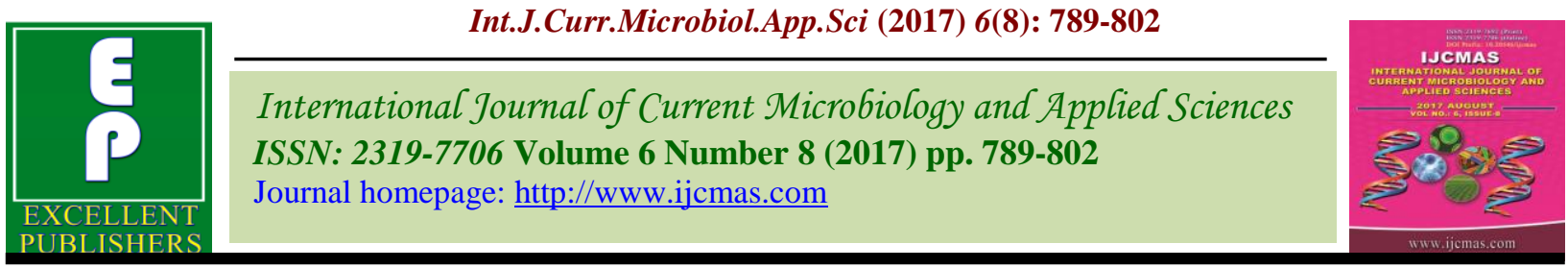

Original Research Article

https://doi.org/10.20546/ijcmas.2017.608.100

\title{
Estimation of Heterosis for Growth, Yield and Quality Traits in Bottle Gourd [Lagenaria siceraria (Mol.) Standl.]
}

\author{
Deepak Kumar Gautam*, G.C. Yadav, Pushpendra Kumar, \\ Vimlesh Kumar and Manvendra Singh
}

Department of Vegetable Science, Narendra Deva University of Agriculture and Technology, Kumarganj, Faizabad-224 229 (U.P.), India

*Corresponding author

\section{A B S T R A C T}

The present investigation was carried out in two different seasons with aim to assess the heterobeltosis and standard heterosis in bottle gourd. The present study was comprised of 45 crosses developed through diallel mating design using 10 parents namely, NDBG-49-2 $\left(\mathrm{P}_{1}\right)$, N. Rashmi $\left(\mathrm{P}_{2}\right)$, N. Prabha $\left(\mathrm{P}_{3}\right)$, N. Pooja $\left(\mathrm{P}_{4}\right)$, Pusa Naveen $\left(\mathrm{P}_{5}\right)$, Pb. Komal $\left(\mathrm{P}_{6}\right)$, NDBG S-1 $\left(\mathrm{P}_{7}\right)$, PBOG-3 $\left(\mathrm{P}_{8}\right)$, NDBG-11 $\left(\mathrm{P}_{9}\right)$ and Faizabadi Local $\left(\mathrm{P}_{10}\right)$ at MES,

\section{Keywords}

Bottle gourd, Heterobeltiosis, Economic heterosis and fruit yield per plant.

\section{Article Info}

\section{Accepted:}

14 June 2017

Available Online:

10 August 2017 Vegetable Science, NDUA\&T, Kumarganj, Faizabad (U.P.) India during kharif 2015 ( $\left.\mathrm{E}_{1}\right)$, and Rabi, 2015-16 ( $\left.\mathrm{E}_{2}\right)$. The experiments were laid out in RBD with three replications having each experimental unit of single row with spacing of $3.0 \mathrm{~m} \times 0.5 \mathrm{~m}$. The observations were recorded on parents and $F_{1}$ 's for eighteen quantitative traits including six quality traits viz days to first staminate flower anthesis, days to first pistillate flower anthesis, node number to first staminate flower, node number to first pistillate flower appearance, days to first fruit harvest, vine length at last picking stage (m), number of primary branches per plant, fruit length $(\mathrm{cm})$, fruit circumference $(\mathrm{cm})$, fruit weight $(\mathrm{kg})$, number of fruits per plant, fruit yield per plant $(\mathrm{kg})$, total soluble solids $\left({ }^{\circ} \mathrm{B}\right)$, ascorbic acid $(\mathrm{mg} / 100 \mathrm{~g}$ fresh fruit), reducing sugar $(\%)$, non-reducing sugar $(\%)$, total sugars $(\%)$ and dry matter content in fruit $(\%)$. The present heterobeltiosis for fruit yield per plant heterosis range from $-3.84\left(\mathrm{P}_{2} \times \mathrm{P}_{7}\right)$ to $64.49 \%\left(\mathrm{P}_{5} \times \mathrm{P}_{8}\right)$ in pooled in over better parent. The best five crosses which showed positive and significant heterosis over better parent were $\mathrm{P}_{3} \times \mathrm{P}_{9}$ (67.26\%), $\mathrm{P}_{5} \times \mathrm{P}_{8}(64.49 \%), \mathrm{P}_{6} \times \mathrm{P}_{9}(63.58 \%), \mathrm{P}_{4} \times \mathrm{P}_{5}(60.66 \%)$ and $\mathrm{P}_{2} \times \mathrm{P}_{4}(58.76 \%)$ Standard heterosis range from were $9.80\left(\mathrm{P}_{4} \times \mathrm{P}_{8}\right)$ to $69.17 \%\left(\mathrm{P}_{3} \times \mathrm{P}_{9}\right)$ in pooled. The best five crosses which showed desirable and significant positive standard heterosis in the pooled analysis were $\mathrm{P}_{3} \times \mathrm{P}_{9}(69.17 \%), \mathrm{P}_{6} \times \mathrm{P}_{9}(65.45 \%), \mathrm{P}_{5} \times \mathrm{P}_{8}(64.49 \%), \mathrm{P}_{4} \times \mathrm{P}_{5}(60.66$ $\%$ ) and $\mathrm{P}_{7} \times \mathrm{P}_{9}$ (56.80) for fruit yield per plant indicating that there was a great scope of realizing higher yield in bottle gourd through heterosis breeding.

\section{Introduction}

Bottle gourd [Lagenaria siceraria (Mol.) Standl, $2 \mathrm{n}=2 \mathrm{x}=22]$ is an important cultivated annual cucurbitaceous crop grown throughout the country. Being warm season vegetable crop it thrives well in warm and humid climate but at present it's off season cultivation has progressively stretched throughout the year in northern Indian plains. According to De Candolle (1882), bottle gourd has been found in wild form in South Africa and India. However, Cutler and Whitaker (1961) are of the view that probably 
it is indigenous to tropical Africa on the basis of variability in seeds and fruits. The tender fruits of bottle gourd can be used as a vegetable or for making sweets (e.g. Halva, kheer, petha and burfi etc.), kofta and pickles. The fruit is rich in pectin also, which showed good prospects for jelly preparation. A decoction made from the leaf is a very good medicine for jaundice. The fruit has cooling effect, it is a cardiotonic and diuretic, good for people suffering from biliousness, indigestion and convalescences i.e., regain health after illness. The pulp is good for overcoming constipation, cough, night blindness and as an antidote against certain poisons. The plant extract is used as a cathartic and seeds are used in dropsy. In addition, the seeds and seed oil are edible. The fruits contain 96.3 per cent moisture, 2.9 per cent carbohydrate, 0.2 per cent protein, 0.1 per cent fat, 0.5 per cent mineral matter and $11 \mathrm{mg}$ of vitamin C (Ascorbic acid) per $100 \mathrm{~g}$ fresh weight (Thamburaj and Singh, 2005). The exploitation of heterosis is much easier in cross-pollinated vegetable crops. Bottle gourd being monoecious provides ample scope for the utilization of the hybrid vigour for yield improvement of this crop. The selection of suitable parents for hybridization on the basis of phenotypic performance alone is not a sound procedure as phenotypically superior lines may yield poor recombinants in the segregating generations. It is, therefore, essential that parents should be selected on the basis of their genetic potential.

\section{Materials and Methods}

The experimental materials consisted of 10 promising parental lines of bottle gourd and their $\mathrm{F}_{1}$ progenies. The selected parental lines i.e. NDBG-49-2 $\left(\mathrm{P}_{1}\right), \mathrm{N}$. Rashmi $\left(\mathrm{P}_{2}\right), \mathrm{N}$. Prabha $\left(\mathrm{P}_{3}\right)$, N. Pooja $\left(\mathrm{P}_{4}\right)$, Pusa Naveen $\left(\mathrm{P}_{5}\right)$, $\mathrm{Pb}$. Komal $\left(\mathrm{P}_{6}\right)$, NDBG S-1 $\left(\mathrm{P}_{7}\right)$, PBOG-3 $\left(\mathrm{P}_{8}\right)$, NDBG-11 $\left(\mathrm{P}_{9}\right)$ and Faizabadi Local $\left(\mathrm{P}_{10}\right)$ were crossed in the all possible combinations, excluding reciprocals. At NDUA\&T,
Kumarganj, Faizabad (U.P.). These experimental materials were grown under Randomized Block Design (RBD) with three replications at Main Experiment Station, Department of Vegetable Science, Narendra Deva University of Agriculture and Technology, Narendra Nagar, Kumarganj, Faizabad (U.P.) India. The treatments were sown in rows spaced 3.0 meters apart with a plant to plant spacing of 0.5 meter. The experiments were laid out during the Kharif season of 2015-16 and off season in winter 2015-16 seeds for the study of heterosis. All the recommended agronomic package of practices and protection measures were followed to raise a good crop. Fertilizers and manures were applied as per recommended dose. Observations were recorded on all the six plants maintained carefully in each plot for eighteen quantitative characters viz., days to first staminate flower anthesis, days to first pistillate flower anthesis, node number to first staminate flower, node number to first pistillate flower appearance, days to first fruit harvest, vine length at last picking stage (m), number of primary branches per plant, fruit length $(\mathrm{cm})$, fruit circumference $(\mathrm{cm})$, fruit weight $(\mathrm{kg})$, number of fruits per plant, fruit yield per plant $(\mathrm{kg})$, total soluble solids $\left({ }^{\circ} \mathrm{B}\right)$, ascorbic acid (mg/100 $\mathrm{g}$ fresh fruit), reducing sugar (\%), non-reducing sugar (\%), total sugars (\%) and dry matter content in fruit (\%). Analysis of variance was carried out as suggested by Hayes et al., (1955).

\section{Results and Discussion}

The exploitation of heterosis refers as the superiority of $F_{1}$ hybrid over its parent in terms of yield and its attributing traits. The exploitation of heterosis requires an intensive evaluation of germplasm to find out diverse donors with high nicking of genes and further identification of heterotic crosses. In the present study the estimates of heterosis over better parent (BP) and standard variety (SV) Pusa Naveen were calculated for fifteen $F_{1}$ 's 
during both the seasons $\left(\mathrm{E}_{1}, \mathrm{E}_{2}\right)$ and over seasons (pooled). Perusal of table 1 revealed that nature and magnitude of heterosis differed for different traits and over seasons in various hybrid combinations. The heterobeltiosis for fruit yield per plant -9.77 $\left(\mathrm{P}_{2} \times \mathrm{P}_{7}\right)$ to $61.89 \%\left(\mathrm{P}_{2} \times \mathrm{P}_{4}\right)$ and $-1.96\left(\mathrm{P}_{3} \times\right.$ $\left.\mathrm{P}_{5}\right)$ to $60.77 \%\left(\mathrm{P}_{3} \times \mathrm{P}_{9}\right)$ in Kharif, $2015\left(\mathrm{E}_{1}\right)$, $3.23\left(\mathrm{P}_{2} \times \mathrm{P}_{7}\right)$ to $99.44 \%\left(\mathrm{P}_{3} \times \mathrm{P}_{9}\right)$ and 15.22 $\left(\mathrm{P}_{4} \times \mathrm{P}_{8}\right)$ to $80.05 \%\left(\mathrm{P}_{3} \times \mathrm{P}_{9}\right)$ in Rabi, 2015$16\left(\mathrm{E}_{2}\right)$ and $-3.84\left(\mathrm{P}_{2} \times \mathrm{P}_{7}\right)$ to $64.49 \%\left(\mathrm{P}_{5} \mathrm{x}\right.$ $\left.\mathrm{P}_{8}\right)$ and $9.80\left(\mathrm{P}_{4} \times \mathrm{P}_{8}\right)$ to $69.17 \%\left(\mathrm{P}_{3} \times \mathrm{P}_{9}\right)$ in pooled. A wide range of variations in positive and negative direction of heterosis were also recorded for remaining traits during both the seasons and pooled. A perusal of tables 1 and 2 revealed that crosses exhibiting significant and positive estimates of heterosis for one or both types of heterosis for fruit yield also exhibited significant heterosis for some other important yield and yield attributing traits. In contrast none of the crosses showed significant and desirable heterosis for all the traits. The above results are in conformity with the findings of Sirohi et al., (1985), Kumar et al., (1998) Dubey and Maurya (2003) and Ghuge, et al., (2016).

For maturity traits negative heterosis is desirable. Since hybrids with heterosis for earliness produce first fruit earlier as compared to parents, thereby increasing their productivity per day per unit area and as a consequence fetch good prices in the market by early supply of produce. A close examination of heterosis values of five maturity traits viz., days to first staminate and pistillate flower opening, node number to first staminate and pistillate flower opening, node number to first staminate and pistillate flower appearance and days to first fruit harvest, revealed that none of the hybrids for days to first staminate flower anthesis, four and one hybrids for days to first staminate flower anthesis, five and four hybrids for node number to first staminate flower appearance, two and one hybrids for node number to first pistillate flower appearance, and none of the and one hybrids for first fruit harvest fruit in pooled exhibited significant and desirable heterosis in respect to better and standard parent respectively. However, top ranked crosses for fruit yield were almost at par for earliness and thereby showing good scope for early hybrids.

Our study further revealed that at least one parent $\left(\mathrm{P}_{3}, \mathrm{P}_{4} \mathrm{P}_{5}, \mathrm{P}_{6}, \mathrm{P}_{7}, \mathrm{P}_{8}\right.$ and $\left.\mathrm{P}_{9}\right)$ with early maturity was invariably involved in the five top ranked $\mathrm{F}_{1}$ hybrids $\left(\mathrm{P}_{3} \times \mathrm{P}_{9}, \mathrm{P}_{6} \times \mathrm{P}_{9}, \mathrm{P}_{5} \mathrm{x}\right.$ $\mathrm{P}_{8}, \mathrm{P}_{4} \times \mathrm{P}_{5}$ and $\mathrm{P}_{7} \times \mathrm{P}_{9}$ ) for fruit yield over standard parent in pooled. Further the earliness of parent as well as crosses were directly associated with the crosses having high magnitude of heterosis. It may therefore, safely be concluded that either of parents, $\mathrm{P}_{3}$, $\mathrm{P}_{4} \mathrm{P}_{5}, \mathrm{P}_{6}, \mathrm{P}_{7}, \mathrm{P}_{8}$ and $\mathrm{P}_{9}$ or any two of them may be a better choice in any heterosis breeding programme intended to breed high yielding hybrids with considerable earliness. The present observations are in agreement with the findings of Singh (2008) and Maurya (2010).

Out of significant crosses for fruit yield, very less crosses showed positive and significant heterobeltiosis for quality traits viz., T.S.S., ascorbic acid, reducing and non-reducing sugar total sugars and dry matter. For instance, out of twenty seven crosses which exhibited significant heterobeltiosis for fruit yield, six crosses for T.S.S., two crosses for ascorbic acid, nine crosses for reducing, twelve crosses for non-reducing sugar and twenty three for dry matter content of fruit showed significant and desirable heterosis. However, the number of crosses which showed significant standard heterosis for quality traits along with fruit yield were generally more in number than the crosses for significant better parent heterosis. This showed negative association for heterosis between fruit yield and quality traits. 
Table.1 Estimates of heterosis (\%) over better parent (BP) and standard variety (SV) Pusa Naveen during two seasons ( $\left.\mathrm{E}_{1}, \mathrm{E}_{2}\right)$ and pooled

\begin{tabular}{|c|c|c|c|c|c|c|c|c|c|c|c|c|}
\hline \multirow{3}{*}{ Crosses } & \multicolumn{6}{|c|}{ Days to first staminate flower anthesis } & \multicolumn{6}{|c|}{ Days to first pistillate flower anthesis } \\
\hline & \multicolumn{2}{|c|}{$\mathbf{E}_{1}$} & \multicolumn{2}{|c|}{$\mathbf{E}_{2}$} & \multicolumn{2}{|c|}{ Pooled } & \multicolumn{2}{|c|}{$\mathbf{E}_{1}$} & \multicolumn{2}{|c|}{$\mathbf{E}_{2}$} & \multicolumn{2}{|c|}{ Pooled } \\
\hline & BP & SV & BP & SV & BP & SV & BP & SV & BP & SV & BP & SV \\
\hline$\overline{\mathbf{P}_{1} \times \mathbf{P}_{2}}$ & $10.16^{* * *}$ & $8.34^{* * *}$ & $9.66^{* * *}$ & $9.33^{* * *}$ & $9.82^{* * *}$ & $9.00^{* *}$ & 4.70 & $11.81^{* *}$ & -3.95 & $-9.19^{* *}$ & -1.94 & -2.20 \\
\hline $\mathbf{P}_{1} \times \mathbf{P}_{3}$ & -4.53 & -0.94 & 3.62 & 4.26 & 0.88 & 2.54 & -4.44 & 2.05 & 2.84 & -1.06 & 0.24 & -0.02 \\
\hline $\mathbf{P}_{1} \times \mathbf{P}_{4}$ & 0.38 & 4.00 & 5.31 & $5.96^{*}$ & 3.60 & 5.31 & 5.19 & $11.65^{* * *}$ & 2.14 & -1.73 & 3.00 & 2.73 \\
\hline $\mathbf{P}_{1} \times \mathbf{P}_{5}$ & 2.04 & 2.04 & 0.25 & 0.25 & 0.84 & 0.84 & $11.96^{* * *}$ & $11.96^{* * *}$ & 0.06 & -3.73 & 1.77 & 1.50 \\
\hline $\mathbf{P}_{1} \times \mathbf{P}_{6}$ & $6.57 *$ & $6.70^{*}$ & 2.54 & 3.17 & 2.64 & 4.33 & $13.01^{* * *}$ & $8.85^{* *}$ & $5.62 *$ & -2.99 & $8.16 * *$ & 0.95 \\
\hline $\mathbf{P}_{1} \times \mathbf{P}_{7}$ & -2.86 & 0.79 & 0.59 & 1.21 & -0.57 & 1.07 & 2.96 & $9.95 * *$ & 5.19 & 1.21 & 4.40 & 4.12 \\
\hline $\mathbf{P}_{1} \times \mathbf{P}_{8}$ & 0.18 & 3.94 & 2.99 & 2.56 & 1.34 & 3.01 & -0.77 & 5.96 & 2.61 & -3.06 & 0.21 & -0.05 \\
\hline $\mathbf{P}_{1} \times \mathbf{P}_{9}$ & $24.48^{* * *}$ & $29.15^{* * *}$ & -0.86 & -0.24 & $7.68^{* * *}$ & $9.46^{* * *}$ & $18.37^{* * *}$ & $26.41^{* *}$ & 2.31 & -1.57 & $8.04 * *$ & $7.75 * *$ \\
\hline $\mathbf{P}_{1} \times \mathbf{P}_{10}$ & $\begin{array}{l}-2.99 \\
\end{array}$ & 0.65 & 0.63 & 1.25 & -0.59 & 1.05 & $-7.62^{*}$ & $\begin{array}{l}-1.34 \\
\end{array}$ & 2.12 & -1.75 & -1.35 & -1.62 \\
\hline $\mathbf{P}_{2} \times \mathbf{P}_{3}$ & 3.23 & 1.53 & $8.10^{* * *}$ & $7.77^{* * *}$ & 6.50 & 5.71 & $-10.38^{* * *}$ & -0.97 & 0.45 & -5.03 & -3.54 & -3.68 \\
\hline $\mathbf{P}_{2} \times \mathbf{P}_{4}$ & 3.79 & 2.08 & $\begin{array}{l}-0.95 \\
\end{array}$ & -1.25 & 0.60 & -0.15 & -2.16 & 3.85 & 0.81 & -4.68 & -1.70 & -1.84 \\
\hline $\mathbf{P}_{2} \times \mathbf{P}_{5}$ & 5.01 & 3.29 & 2.02 & 1.71 & 3.00 & 2.23 & 2.73 & 2.73 & 5.09 & -0.64 & 0.63 & 0.48 \\
\hline $\mathbf{P}_{2} \times \mathbf{P}_{6}$ & 2.17 & 0.48 & -0.20 & -0.50 & 0.58 & -0.17 & 2.38 & -1.39 & -1.54 & $-9.57 * *$ & -0.20 & $-6.85^{*}$ \\
\hline $\mathbf{P}_{2} \times \mathbf{P}_{7}$ & $12.77 * *$ & 10.91 ** & $7.97 * *$ & $7.64 * *$ & $9.54 * *$ & $8.72^{* * *}$ & 2.66 & $13.44 * *$ & -1.48 & $-6.86^{* *}$ & 0.05 & -0.10 \\
\hline $\mathbf{P}_{2} \times \mathbf{P}_{8}$ & 6.08 & 4.33 & 0.80 & 0.37 & 2.44 & 1.68 & 4.17 & $15.11^{* * *}$ & 2.64 & $\begin{array}{l}-3.02 \\
\end{array}$ & 3.16 & 3.01 \\
\hline $\mathbf{P}_{2} \times \mathbf{P}_{9}$ & 5.31 & 3.58 & -0.07 & -0.37 & 1.69 & 0.94 & -0.45 & $9.99 * *$ & $10.05 * *$ & 4.05 & $6.18^{*}$ & $6.03^{*}$ \\
\hline $\mathbf{P}_{2} \times \mathbf{P}_{10}$ & 4.68 & 2.96 & 1.63 & 1.32 & 2.63 & 1.86 & $-5.73 *$ & 4.16 & $11.81^{* * *}$ & $5.72^{*}$ & 5.35 & 5.20 \\
\hline $\mathbf{P}_{3} \times \mathbf{P}_{4}$ & $14.66^{* *}$ & $18.79 * *$ & -3.31 & -2.49 & 1.27 & 4.53 & $7.74 *$ & $14.35^{* * *}$ & $-7.29^{* *}$ & $-5.59 *$ & -2.15 & 1.05 \\
\hline $\mathbf{P}_{3} \times \mathbf{P}_{5}$ & 3.76 & 3.76 & 0.51 & 0.51 & 1.58 & 1.58 & 4.12 & 4.12 & 0.52 & 0.52 & 1.72 & 1.72 \\
\hline $\mathbf{P}_{3} \times \mathbf{P}_{6}$ & 5.21 & 5.33 & 1.90 & 2.76 & 1.63 & 3.61 & 3.23 & -0.57 & $12.03^{* * *}$ & 2.89 & $9.00 * *$ & 1.74 \\
\hline $\mathbf{P}_{3} \times \mathbf{P}_{7}$ & $7.30^{*}$ & $16.31^{* *}$ & -3.37 & -2.55 & 0.32 & 3.67 & 3.40 & $16.76^{* * *}$ & $-6.30^{*}$ & $-5.79^{*}$ & $\begin{array}{l}-2.99 \\
\end{array}$ & 1.72 \\
\hline $\mathbf{P}_{3} \times \mathbf{P}_{8}$ & 2.19 & 8.40 & 1.64 & 1.21 & 1.83 & 3.59 & -1.30 & $10.87 * *$ & 5.20 & $\begin{array}{l}-0.61 \\
\end{array}$ & 2.78 & 3.21 \\
\hline $\mathbf{P}_{3} \times \mathbf{P}_{9}$ & -4.36 & 1.32 & 0.20 & 1.05 & -2.13 & 1.14 & $-10.49^{* *}$ & $\begin{array}{l}-0.85 \\
\end{array}$ & -2.85 & 0.13 & $-5.52^{*}$ & -0.19 \\
\hline $\mathbf{P}_{3} \times \mathbf{P}_{10}$ & -4.63 & 3.38 & 0.81 & 1.66 & -1.07 & 2.23 & $-14.57 * *$ & -3.75 & -2.26 & 0.61 & $-6.61 *$ & -0.84 \\
\hline $\mathbf{P}_{4} \times \mathbf{P}_{5}$ & $7.41^{*}$ & $7.41^{*}$ & 1.45 & 1.45 & 3.41 & 3.41 & -2.00 & -2.00 & -1.99 & -1.99 & $\begin{array}{l}-1.99 \\
\end{array}$ & -1.99 \\
\hline $\mathbf{P}_{4} \times \mathbf{P}_{6}$ & 3.62 & 3.75 & 4.67 & $7.66 * *$ & 4.33 & $6.37 *$ & $8.09 *$ & 4.11 & 10.58 ** & 1.56 & $9.72 * *$ & 2.41 \\
\hline $\mathbf{P}_{4} \times \mathbf{P}_{7}$ & -3.91 & -0.44 & -6.04* & $\begin{array}{l}-3.18 \\
\end{array}$ & -5.33 & \begin{tabular}{l|l|}
-2.28 \\
\end{tabular} & 3.53 & $9.88^{* * *}$ & $-5.93^{*}$ & $-5.41^{*}$ & $\begin{array}{l}-3.47 \\
\end{array}$ & -0.32 \\
\hline $\mathbf{P}_{4} \times \mathbf{P}_{8}$ & 2.64 & 6.34 & 1.60 & 1.17 & 1.13 & 2.87 & 5.65 & $12.13 * *$ & 4.99 & -0.81 & $-7.77 * *$ & 3.50 \\
\hline $\mathbf{P}_{4} \times \mathbf{P}_{9}$ & -4.38 & -0.94 & $-5.26^{*}$ & -2.38 & -4.97 & -1.91 & $-6.79 *$ & $\begin{array}{l}-1.07 \\
\end{array}$ & $-8.29^{* *}$ & $-6.60^{* * *}$ & 2.18 & -4.76 \\
\hline $\mathbf{P}_{4} \times \mathbf{P}_{10}$ & 0.58 & 4.20 & 0.12 & 3.16 & 0.28 & 3.51 & 4.12 & $10.51 * *$ & 1.18 & 3.04 & 5.85 & $5.53^{*}$ \\
\hline $\mathbf{P}_{5} \times \mathbf{P}_{6}$ & $7.01 *$ & $7.01 *$ & 4.22 & 4.22 & 5.14 & 5.14 & 5.86 & 1.96 & $5.85^{*}$ & -2.79 & 3.97 & -1.20 \\
\hline $\mathbf{P}_{5} \times \mathbf{P}_{7}$ & $21.85^{* *}$ & $21.85^{* * *}$ & -2.57 & -2.57 & 5.49 & 5.49 & $23.29^{* * *}$ & $23.29^{* * *}$ & $-5.68^{*}$ & $-5.68^{*}$ & 1.42 & 3.97 \\
\hline $\mathbf{P}_{5} \times \mathbf{P}_{8}$ & 2.74 & 2.74 & 4.90 & 4.46 & 3.89 & 3.89 & -1.65 & -1.65 & $8.97 * *$ & 2.95 & 2.02 & 1.42 \\
\hline $\mathbf{P}_{5} \times \mathbf{P}_{9}$ & 0.40 & 0.40 & 4.39 & 4.39 & 3.07 & 3.07 & 0.25 & 0.25 & 2.91 & 2.91 & -1.36 & 2.02 \\
\hline $\mathbf{P}_{5} \times \mathbf{P}_{10}$ & $12.11 * *$ & $12.11 * *$ & 2.19 & 2.19 & 5.46 & 5.46 & $7.01 *$ & $7.01 *$ & $-5.54 *$ & $-5.54 *$ & $10.00^{* * *}$ & -1.36 \\
\hline $\mathbf{P}_{6} \times \mathbf{P}_{7}$ & 10.08 ** & $10.21 * *$ & -2.89 & -0.12 & 1.32 & 3.29 & $16.94 * *$ & $12.63^{* *}$ & $6.37^{*}$ & -2.31 & 2.58 & 2.67 \\
\hline $\mathbf{P}_{6} \times \mathbf{P}_{8}$ & $7.99 *$ & $8.12^{*}$ & 3.71 & 3.28 & 3.10 & 4.88 & 4.52 & 0.67 & 1.57 & $-6.72^{* *}$ & $7.75^{* * *}$ & -4.26 \\
\hline $\mathbf{P}_{6} \times \mathbf{P}_{9}$ & $8.55 * *$ & $8.68 * *$ & 0.86 & 3.73 & 3.35 & 5.36 & -0.58 & -4.24 & $12.11^{* * *}$ & 2.97 & $9.36 * *$ & 0.57 \\
\hline $\mathbf{P}_{6} \times \mathbf{P}_{10}$ & 5.99 & 6.11 & -0.92 & 1.91 & 1.32 & 3.29 & $8.17^{*}$ & 4.19 & $9.98 * *$ & 1.01 & -2.66 & 2.07 \\
\hline $\mathbf{P}_{7} \times \mathbf{P}_{8}$ & 2.88 & $9.13^{* *}$ & 4.74 & 4.30 & 4.10 & $5.89^{*}$ & -2.46 & $9.56 * *$ & -2.78 & $-8.15^{* *}$ & -1.25 & -2.25 \\
\hline $\mathbf{P}_{7} \times \mathbf{P}_{9}$ & 5.31 & $11.56 * *$ & 1.45 & $5.67^{*}$ & 2.74 & $7.61^{* * *}$ & -1.10 & $9.56^{* * *}$ & 0.00 & 0.55 & -2.67 & 3.55 \\
\hline $\mathbf{P}_{7} \times \mathbf{P}_{10}$ & 0.36 & $9.64 * *$ & -4.92 & -1.81 & -3.11 & 1.97 & 1.16 & $13.97 * *$ & -4.41 & -3.88 & 4.33 & 2.06 \\
\hline $\mathbf{P}_{8} \times \mathbf{P 9}$ & $6.75^{*}$ & $13.08^{* * *}$ & 4.50 & 4.06 & 5.23 & $7.04 *$ & -0.37 & $10.37 * *$ & $7.94 * *$ & 1.98 & -0.13 & 4.77 \\
\hline $\mathbf{P}_{8} \times \mathbf{P}_{10}$ & 5.22 & $11.62^{* * *}$ & $8.10^{* * *}$ & $7.65^{* * *}$ & $7.11^{*}$ & $8.96^{* * *}$ & -2.58 & $9.42 * *$ & 1.33 & -4.26 & 0.54 & 0.29 \\
\hline $\mathbf{P}_{9} \times \mathbf{P}_{10}$ & $7.59 *$ & $13.98^{* * *}$ & 0.15 & 3.43 & 2.07 & $6.91 *$ & 2.28 & $13.29 * *$ & -0.26 & 2.66 & -7.77 & $6.20^{*}$ \\
\hline $\begin{array}{c}\text { No. of crosses with } \\
\text { significant }(+) \text { heterosis }\end{array}$ & 15 & 19 & 4 & 7 & 4 & 9 & 9 & 24 & 10 & 1 & 8 & 4 \\
\hline $\begin{array}{c}\text { No. of crosses with } \\
\text { significant (-) 'heterosis }\end{array}$ & 0 & 0 & 2 & o & 0 & 0 & 5 & 0 & 5 & 11 & 4 & 1 \\
\hline Range of heterosis & -4.63 to 21.85 & -.94 to 29.15 & $\begin{array}{l}-6.04 \text { to } 9.66 \\
\end{array}$ & -2.57 to 9.33 & -5.33 to 9.82 & -2.28 to 9.46 & -14.57 to 23.29 & 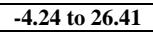 & -8.29 to 12.11 & -9.57 to 5.72 & -7.77 to 10.00 & -6.85 to 7.75 \\
\hline
\end{tabular}
$*, * *$ Significant at 5 per cent and 1 per cent probability levels, respectively. 
Table.1 Cont.....

\begin{tabular}{|c|c|c|c|c|c|c|c|c|c|c|c|c|}
\hline \multirow{3}{*}{ Crosses } & \multicolumn{6}{|c|}{ Node number to first staminate flower appearance } & \multicolumn{6}{|c|}{ Node number to first pistillate flower appearance } \\
\hline & \multirow{2}{*}{\multicolumn{2}{|c|}{$\mathbf{E}_{1}$}} & \multirow{2}{*}{\multicolumn{2}{|c|}{\begin{tabular}{ll|l}
\multicolumn{3}{c|}{$\mathbf{E}_{2}$} \\
$\mathrm{BP}$ & $\mathrm{SV}$ \\
\end{tabular}}} & \multicolumn{2}{|c|}{ Pooled } & \multirow{2}{*}{\multicolumn{2}{|c|}{$\mathbf{E}_{1}$}} & \multirow{2}{*}{\multicolumn{2}{|c|}{$\mathrm{E}_{2}$}} & \multicolumn{2}{|c|}{ Pooled } \\
\hline & & SV & & & BP & SV & & SV & & SV & BP & SV \\
\hline $\mathbf{P}_{1} \times \mathbf{P}_{2}$ & -0.39 & $-18.36 * *$ & $29.63^{* * *}$ & $37.21^{* * *}$ & 10.99 & 0.98 & $26.48^{* * *}$ & $84.26 * *$ & $18.13^{*}$ & -2.51 & $23.60^{* *}$ & $42.45 * *$ \\
\hline $\mathbf{P}_{1} \times \mathbf{P}_{3}$ & -0.54 & $-18.49 * *$ & $14.45^{* * *}$ & $35.06^{* * *}$ & -0.70 & 0.15 & 5.90 & $89.02 * *$ & $45.36 * *$ & $12.45^{*}$ & $17.23^{* * *}$ & $52.12 * *$ \\
\hline$\overline{P_{1} \times \mathbf{P}_{4}}$ & 11.41 & -8.69 & $36.11^{* * *}$ & $52.18^{* * *}$ & $11.54 *$ & $12.49^{*}$ & $-24.13^{* * *}$ & $71.85^{* *}$ & $50.74 * *$ & 10.97 & -6.91 & $42.52 * *$ \\
\hline $\mathbf{P}_{1} \times \mathbf{P}_{5}$ & $16.73^{* * *}$ & -4.33 & -0.36 & -0.36 & -2.95 & -2.95 & $32.08^{* *}$ & $32.08 * *$ & -9.96 & $-12.80^{*}$ & 10.46 & 10.46 \\
\hline $\mathbf{P}_{1} \times \mathbf{P}_{6}$ & 5.06 & $-14.09 * *$ & $-28.32 * *$ & -2.33 & $-10.76^{*}$ & -10.00 & $46.93 * *$ & $68.51^{* *}$ & -4.01 & $\begin{array}{l}-7.04 \\
\end{array}$ & $18.03^{* *}$ & $32.11^{* * *}$ \\
\hline $\mathbf{P}_{1} \times \mathbf{P}_{7}$ & 5.47 & $-15.37 * *$ & $29.33^{* *}$ & $75.13^{* *}$ & $16.77 * *$ & $16.12^{* *}$ & $8.40^{*}$ & $112.13^{* *}$ & $70.62^{* * *}$ & $24.61 * *$ & $24.43^{* * *}$ & $69.95^{* *}$ \\
\hline $\mathbf{P}_{1} \times \mathbf{P}_{8}$ & $25.74 * *$ & 3.05 & $10.73^{*}$ & $17.53^{* * *}$ & 7.18 & 8.09 & $28.54 * *$ & $152.91 * *$ & $19.62 * *$ & 7.14 & $25.89^{* * *}$ & $82.67 * *$ \\
\hline $\mathbf{P}_{1} \times \mathbf{P}_{9}$ & $20.50^{* * *}$ & -1.24 & -4.41 & $12.52^{* * *}$ & 4.94 & 3.55 & $47.87 * *$ & $131.58^{* * *}$ & 13.22 & 2.41 & $35.76^{* * *}$ & $69.34 * *$ \\
\hline $\mathbf{P}_{1} \times \mathbf{P}_{10}$ & 7.53 & $-11.87^{*}$ & 0.13 & $35.60 * *$ & 3.76 & 4.65 & $-60.70^{* * *}$ & $-23.75^{* * *}$ & \begin{tabular}{|l|}
-1.68 \\
\end{tabular} & $-13.83^{*}$ & $-43.24 * *$ & $-18.97 * *$ \\
\hline $\mathbf{P}_{2} \times \mathbf{P}_{3}$ & 5.86 & $-12.09^{*}$ & $44.85^{* * *}$ & $53.31^{* * *}$ & $21.64 * *$ & $10.66^{*}$ & 3.02 & $50.07 * *$ & $49.81^{* * *}$ & $15.90^{*}$ & $15.93^{*}$ & $33.60^{* * *}$ \\
\hline $\mathbf{P}_{2} \times \mathbf{P}_{4}$ & -9.16 & $-24.56^{* * *}$ & $27.61^{* * *}$ & $35.06^{* * *}$ & 5.72 & $\begin{array}{l}-3.82 \\
\end{array}$ & 9.02 & $58.81 * *$ & $27.61^{* * *}$ & -6.05 & $\begin{array}{l}10.68 \\
\end{array}$ & $27.56^{* * *}$ \\
\hline $\mathbf{P}_{2} \times \mathbf{P}_{5}$ & $21.07^{* * *}$ & 0.54 & $53.67^{* * *}$ & $53.67^{* *}$ & $30.83^{* * *}$ & $19.02 * *$ & $38.31^{* * *}$ & $38.31 * *$ & $29.46^{* * *}$ & 6.84 & $23.14^{* * *}$ & $23.14 * *$ \\
\hline $\mathbf{P}_{2} \times \mathbf{P}_{6}$ & $24.28 * *$ & 1.62 & 9.86 & $16.28 * *$ & $17.31^{* * *}$ & 6.72 & $28.73^{* * *}$ & $47.64 * *$ & $24.27 * *$ & 2.56 & 12.50 & $25.92 * *$ \\
\hline $\mathbf{P}_{2} \times \mathbf{P}_{7}$ & $22.88^{* * *}$ & -1.40 & -4.17 & 1.43 & 9.46 & -0.41 & $20.20 * *$ & $75.10^{* * *}$ & $48.05^{* * *}$ & 8.12 & $23.93 * *$ & $42.83^{* *}$ \\
\hline $\mathbf{P}_{2} \times \mathbf{P}_{8}$ & $26.48^{* * *}$ & 5.03 & $33.18^{* * *}$ & $40.97^{* * *}$ & $29.19^{* * *}$ & $17.53 * *$ & $34.78^{* * *}$ & $96.34 * *$ & $27.85^{* *}$ & 5.51 & 32.39 ** & $52.57 * *$ \\
\hline $\mathbf{P}_{2} \times \mathbf{P}_{9}$ & $39.16^{* * *}$ & $15.56^{* * *}$ & -4.34 & 1.25 & $21.55^{* * *}$ & $10.58^{*}$ & $58.66 * *$ & $131.12^{* * *}$ & 7.27 & -11.47 & $40.93^{* *}$ & $62.41^{* * *}$ \\
\hline $\mathbf{P}_{2} \times \mathbf{P}_{10}$ & $28.62^{* * *}$ & 6.81 & $11.55^{*}$ & $18.07^{* * *}$ & $21.71^{* * *}$ & $10.73^{*}$ & $14.67^{* * *}$ & $67.05^{* *}$ & 11.15 & -8.27 & $13.46^{*}$ & $30.76^{* * *}$ \\
\hline $\mathbf{P}_{3} \times \mathbf{P}_{4}$ & $-10.45^{*}$ & $-11.10^{*}$ & $16.11^{* *}$ & $29.82^{* * *}$ & -0.48 & 3.13 & -3.33 & $72.54 * *$ & $42.11^{* * *}$ & 4.63 & 7.75 & $39.82^{* * *}$ \\
\hline $\mathbf{P}_{3} \times \mathbf{P}_{5}$ & 5.15 & 5.15 & $13.06 *$ & $13.06^{* * *}$ & 7.90 & 7.90 & $40.82 * *$ & $40.82 * *$ & 7.06 & $-17.18^{* * *}$ & 12.88 & 12.88 \\
\hline $\mathbf{P}_{3} \times \mathbf{P}_{6}$ & -2.65 & $-20.39 * *$ & $13.09^{* * *}$ & $33.45^{* * *}$ & -7.64 & -1.66 & $24.86^{* * *}$ & $43.20^{* * *}$ & $59.86^{* * *}$ & $23.67^{* * *}$ & $19.53^{* * *}$ & $33.79 * *$ \\
\hline $\mathbf{P}_{3} \times \mathbf{P}_{7}$ & -8.45 & $-26.54 * *$ & 0.35 & $18.43^{* *}$ & $-10.39^{*}$ & $-10.89^{*}$ & $19.49 * *$ & $113.27 * *$ & $50.07 * *$ & 9.60 & $25.86^{* * *}$ & $63.32 * *$ \\
\hline $\mathbf{P}_{3} \times \mathbf{P}_{8}$ & -2.45 & 3.98 & $44.94^{* * *}$ & $53.85^{* * *}$ & $13.64 * *$ & $21.33 * *$ & $10.92^{*}$ & $97.99 * *$ & $20.17^{* * *}$ & -7.04 & $13.58^{*}$ & $47.38^{* * *}$ \\
\hline $\mathbf{P}_{3} \times \mathbf{P}_{9}$ & $86.41 * *$ & $65.00^{* * *}$ & $15.25^{* *}$ & $35.66 * *$ & $56.88^{* * *}$ & $54.79 * *$ & $26.77^{* * *}$ & $98.54 * *$ & $25.19 * *$ & -3.15 & $19.89^{* * *}$ & $49.54 * *$ \\
\hline $\mathbf{P}_{3} \times \mathbf{P}_{10}$ & 3.80 & 5.25 & $30.22^{* * *}$ & $53.67^{* * *}$ & $10.43^{*}$ & $22.10^{* * *}$ & $-10.79^{*}$ & $59.22 * *$ & $41.79^{* * *}$ & 9.69 & 4.31 & $35.36^{* * *}$ \\
\hline $\mathbf{P}_{4} \times \mathbf{P}_{5}$ & $-28.72^{* * *}$ & $-29.24 * *$ & $35.42 * *$ & $35.42^{* *}$ & -6.74 & -6.74 & 8.01 & 8.01 & $36.70^{* * *}$ & 0.64 & 4.46 & 4.46 \\
\hline $\mathbf{P}_{4} \times \mathbf{P}_{6}$ & $19.49^{* * *}$ & -2.29 & $19.36^{* * *}$ & $33.45^{* *}$ & 6.29 & $10.15^{*}$ & $34.28 * *$ & $54 * *$ & $43.32 * *$ & 5.51 & $16.72^{*}$ & $30.64 * *$ \\
\hline $\mathbf{P}_{4} \times \mathbf{P}_{7}$ & $-34.73^{* * *}$ & $-47.63 * *$ & $23.04 * *$ & $37.57 * *$ & $-17.53 * *$ & $-17.99^{* *}$ & $-34.85 * *$ & $27.51^{* *}$ & $28.10^{* * *}$ & -6.45 & $-18.63^{* * *}$ & 11.15 \\
\hline $\mathbf{P}_{4} \times \mathbf{P}_{8}$ & $15.19^{* * *}$ & $14.35 * *$ & 2.64 & 8.94 & 8.53 & $12.47^{*}$ & $14.38^{* * *}$ & $125.03 * *$ & 7.02 & $-21.21 * *$ & 6.52 & $54.56^{* * *}$ \\
\hline $\mathbf{P}_{4} \times \mathbf{P}_{9}$ & $14.63^{* * *}$ & 1.46 & $19.89^{* * *}$ & $34.05^{* *}$ & $14.32^{* * *}$ & $12.80^{*}$ & $19.17^{* * *}$ & $86.64 * *$ & $27.94 * *$ & -5.81 & 13.92 & $42.09 * *$ \\
\hline $\mathbf{P}_{4} \times \mathbf{P}_{10}$ & 9.84 & 9.04 & $16.48^{* * *}$ & $30.23^{* * *}$ & $12.33^{*}$ & $16.41^{* * *}$ & 7.88 & $109.29 * *$ & 12.43 & $-17.22^{* * *}$ & 3.90 & $48.33^{* * *}$ \\
\hline $\mathbf{P}_{5} \times \mathbf{P}_{6}$ & $21.17^{* * *}$ & -0.92 & $39.36^{* * *}$ & $39.36^{* * *}$ & $13.09^{*}$ & $13.09^{*}$ & 19.73* & $19.73^{*}$ & \begin{tabular}{|l|}
-1.53 \\
\end{tabular} & $\begin{array}{l}-1.53 \\
\end{array}$ & 9.49 & 9.49 \\
\hline $\mathbf{P}_{5} \times \mathbf{P}_{7}$ & $-34.50^{* * *}$ & $-47.44 * *$ & $33.63^{* * *}$ & $33.63^{* * *}$ & $-18.78 * *$ & $-19.23^{* *}$ & 13.32 & 13.32 & $27.29 * *$ & -7.04 & 3.51 & 3.51 \\
\hline $\mathbf{P}_{5} \times \mathbf{P}_{8}$ & -2.26 & -2.26 & $48.24^{* * *}$ & $48.24^{* * *}$ & $15.31^{* * *}$ & $15.31^{* * *}$ & $46.41^{* * *}$ & $46.41^{* * *}$ & 3.90 & $\begin{array}{l}-6.94 \\
\end{array}$ & $20.70^{* * *}$ & $20.70^{* * *}$ \\
\hline $\mathbf{P}_{5} \times \mathbf{P}_{9}$ & 3.56 & -8.34 & $13.06^{*}$ & $13.06^{*}$ & 0.44 & -0.89 & $28.10^{* * *}$ & $28.10^{* * *}$ & -2.77 & -12.06 & 8.75 & 8.75 \\
\hline $\mathbf{P}_{5} \times \mathbf{P}_{10}$ & $-12.06^{*}$ & $-12.06^{*}$ & $35.60^{* * *}$ & $35.60^{* * *}$ & 4.52 & 4.52 & $19.73^{*}$ & $19.73^{*}$ & $17.01^{*}$ & 2.56 & 11.45 & 11.45 \\
\hline $\mathbf{P}_{6} \times \mathbf{P}_{7}$ & $-27.32^{* * *}$ & $-41.68 * *$ & $-18.80^{* * *}$ & 9.96 & $-23.28 * *$ & $-23.71 * *$ & 2.79 & $17.89 *$ & $22.44 * *$ & -10.58 & -6.93 & 4.17 \\
\hline $\mathbf{P}_{6} \times \mathbf{P}_{8}$ & $13.54 *$ & -7.16 & $23.31^{* *}$ & $30.89^{* *}$ & -0.37 & 6.08 & $23.62^{* * *}$ & $41.78^{* *}$ & 12.03 & 0.34 & 8.83 & $21.82 * *$ \\
\hline $\mathbf{P}_{6} \times \mathbf{P}_{9}$ & $19.46 * *$ & -2.32 & $10.69 *$ & $30.29 * *$ & $10.49^{*}$ & 9.02 & $50.28^{* *}$ & $72.36 * *$ & -5.22 & $-14.27 *$ & $16.69^{*}$ & $30.61^{* * *}$ \\
\hline $\mathbf{P}_{6} \times \mathbf{P}_{10}$ & $13.54 *$ & -7.16 & $12.29^{* * *}$ & $52.06^{* * *}$ & 6.55 & $13.44 * *$ & $24.22^{* * *}$ & $42.47 * *$ & -4.38 & $-16.19^{* * *}$ & 2.03 & 14.20 \\
\hline $\mathbf{P}_{7} \times \mathbf{P}_{8}$ & 5.59 & $-15.27 * *$ & $23.82^{* * *}$ & $31.43^{* *}$ & 1.54 & 0.98 & 8.49* & $112.31^{* *}$ & $17.59^{*}$ & $-14.12^{*}$ & $10.83^{*}$ & $51.39 * *$ \\
\hline $\mathbf{P}_{7} \times \mathbf{P}_{9}$ & $17.76^{* * *}$ & -5.50 & $25.84^{* * *}$ & $48.12^{* *}$ & $14.68 * *$ & $13.15^{*}$ & $24.81 * *$ & $95.47 * *$ & $52.29 * *$ & 11.22 & $24.16^{* *}$ & $54.87 * *$ \\
\hline $\mathbf{P}_{7} \times \mathbf{P}_{10}$ & $14.99^{*}$ & $\begin{array}{l}-7.73 \\
\end{array}$ & $-13.08^{* * *}$ & $17.71 * *$ & 1.69 & 1.12 & 1.75 & $97.39^{* *}$ & $47.51 * *$ & 7.73 & $12.88^{*}$ & $54.19 * *$ \\
\hline $\mathbf{P}_{8} \times \mathbf{P 9}$ & 12.40* & -0.51 & 9.04 & $15.74^{* * *}$ & 6.56 & 5.15 & $17.53^{* * *}$ & $84.07 * *$ & $23.46^{* * *}$ & 10.58 & $19.18^{*}$ & $48.66^{* * *}$ \\
\hline $\mathbf{P}_{8} \times \mathbf{P}_{10}$ & $9.85^{*}$ & $11.39 *$ & $31.80^{* * *}$ & $39.89^{* *}$ & $13.62^{* * *}$ & $21.31^{* * *}$ & 1.56 & $97.03 * *$ & 11.79 & -2.02 & 4.58 & $49.30 * *$ \\
\hline $\mathbf{P}_{9} \times \mathbf{P}_{10}$ & $14.59 *$ & 1.43 & -5.78 & $10.91^{*}$ & $\begin{array}{r}6.14 \\
\end{array}$ & 4.73 & 10.32 & $72.77 * *$ & $27.85^{* * *}$ & 12.06 & $15.06 *$ & $43.51 * *$ \\
\hline $\begin{array}{c}\text { No. of crosses with } \\
\text { significant }(+) \text { heterosis }\end{array}$ & 22 & 4 & 32 & 39 & 18 & 19 & 30 & 42 & 29 & 4 & 24 & 34 \\
\hline $\begin{array}{c}\text { No. of crosses with } \\
\text { significant (-) 'heterosis }\end{array}$ & 6 & 15 & 3 & $\mathbf{0}$ & 5 & 4 & 4 & 1 & $\mathbf{0}$ & 8 & 2 & 1 \\
\hline Range of heterosis & -34.73 to 86.41 & -47.63 to 65.00 & -28.32 to 53.67 & -2.33 to 75.13 & -23.28 to 56.88 & -23.71 to 54.79 & -60.70 to 47.87 & \begin{tabular}{|l|}
-23.75 to 125.03 \\
\end{tabular} & -9.96 to 70.62 & -21.21 to 24.61 & -43.24 to 40.93 & -18.97 to 82.67 \\
\hline
\end{tabular}

*, ** Significant at 5 per cent and 1 per cent probability levels, respectively. 
Table. 1 Cont....

\begin{tabular}{|c|c|c|c|c|c|c|c|c|c|c|c|c|}
\hline \multirow{3}{*}{ Crosses } & \multicolumn{6}{|c|}{ Days to first fruit harvest } & \multicolumn{6}{|c|}{ Vine length $(\mathbf{m}$} \\
\hline & \multicolumn{2}{|c|}{$\mathbf{E}_{1}$} & \multicolumn{2}{|l|}{ BP } & \multicolumn{2}{|c|}{ Pooled } & \multicolumn{2}{|c|}{$\mathbf{E}_{1}$} & \multicolumn{2}{|c|}{$\mathbf{E}_{2}$} & \multicolumn{2}{|c|}{ Pooled } \\
\hline & BP & SV & BP & SV & BP & SV & BP & SV & BP & SV & BP & SV \\
\hline $\mathbf{P}_{1} \times \mathbf{P}_{2}$ & 1.03 & $7.28^{* *}$ & -3.96 & -4.60 & -2.36 & -0.79 & 8.15 & $39.16^{* *}$ & $-35.11^{* * *}$ & $-49.42^{* * *}$ & -7.82 & -5.73 \\
\hline $\mathbf{P}_{1} \times \mathbf{P}_{3}$ & -5.03 & 0.85 & 3.88 & 4.73 & 0.93 & 3.49 & 0.00 & $28.67 * *$ & -6.40 & $-28.32 * *$ & -2.43 & -0.21 \\
\hline $\mathbf{P}_{1} \times \mathbf{P}_{4}$ & 1.82 & $5.68 *$ & -0.55 & 0.26 & -0.53 & 1.99 & -10.38 & 15.31 & $27.87 * *$ & 1.50 & 5.90 & 8.31 \\
\hline $\mathbf{P}_{1} \times \mathbf{P}_{5}$ & $9.85 * *$ & $9.85^{* * *}$ & 0.30 & 0.30 & 3.35 & 3.35 & -11.58 & 13.78 & $-20.42^{* * *}$ & $-20.42^{* * *}$ & -5.70 & -3.55 \\
\hline $\mathbf{P}_{1} \times \mathbf{P}_{6}$ & $10.16^{\text {*** }}$ & $6.47^{*}$ & $7.36 * *$ & 2.26 & $8.27 * *$ & 3.60 & $-17.66 * *$ & $25.87^{* * *}$ & $-41.30 * *$ & $-49.69 * *$ & $-26.30^{* * *}$ & -12.42 \\
\hline $\mathbf{P}_{1} \times \mathbf{P}_{7}$ & 0.38 & $6.60^{*}$ & 1.72 & 2.56 & 1.28 & 3.85 & $-17.77 * *$ & $42.03 * *$ & $-49.42 * *$ & $-61.27 * *$ & $-15.28^{*}$ & -10.31 \\
\hline $\mathbf{P}_{1} \times \mathbf{P}_{8}$ & -0.83 & 5.31 & 0.01 & -0.07 & -0.54 & 1.65 & $24.14 * *$ & $90.98 * *$ & $-44.26 * *$ & $-40.84 * *$ & -4.23 & $24.18^{* * *}$ \\
\hline $\mathbf{P}_{1} \times \mathbf{P}_{9}$ & $11.65 * *$ & $18.56^{* * *}$ & 1.64 & 2.47 & 4.96 & $7.62 * *$ & $41.64 * *$ & $93.15 * *$ & $-68.94 * *$ & $-51.06 * *$ & $-18.38^{* * *}$ & $20.08^{* * *}$ \\
\hline $\mathbf{P}_{1} \times \mathbf{P}_{10}$ & $-7.36^{* *}$ & -1.62 & -0.57 & 0.24 & -2.82 & -0.35 & 8.25 & $46.85 * *$ & $-12.77 * *$ & $-13.48^{* *}$ & -0.77 & $16.28^{*}$ \\
\hline $\mathbf{P}_{2} \times \mathbf{P}_{3}$ & $-7.29^{* *}$ & -1.35 & 0.63 & -0.04 & -2.02 & -0.46 & $75.18^{* * *}$ & $72.73^{* *}$ & $-13.62 * *$ & $-32.68 * *$ & $40.10^{* * *}$ & $19.32^{* * *}$ \\
\hline $\mathbf{P}_{2} \times \mathbf{P}_{4}$ & -1.01 & 2.73 & -2.21 & -2.85 & -2.62 & -1.07 & $40.63^{* *}$ & $34.34 * *$ & -1.03 & $-21.44 * *$ & $21.45^{* *}$ & 6.07 \\
\hline $\mathbf{P}_{2} \times \mathbf{P}_{5}$ & 1.35 & 1.35 & 1.40 & 0.73 & 0.93 & 0.93 & 17.90 & 17.90 & $-21.44 * *$ & $-21.44 * *$ & -2.04 & -2.04 \\
\hline $\mathbf{P}_{2} \times \mathbf{P}_{6}$ & 1.16 & -2.23 & -2.40 & $-7.04 * *$ & -1.25 & $-5.50^{*}$ & -6.45 & $43.01 * *$ & $16.92^{* *}$ & 0.20 & 2.09 & $21.32 * *$ \\
\hline $\mathbf{P}_{2} \times \mathbf{P}_{7}$ & 2.56 & $9.13^{* *}$ & -3.25 & -3.89 & -1.30 & 0.28 & 0.24 & $73.15^{* *}$ & $30.13^{* * *}$ & 1.43 & $29.23^{* * *}$ & $36.81^{* * *}$ \\
\hline $\mathbf{P}_{2} \times \mathbf{P}_{8}$ & 3.78 & $10.43^{* * *}$ & 0.85 & 0.19 & 1.84 & 3.47 & -7.86 & $41.75 * *$ & $-37.33 * *$ & $-33.49 * *$ & $-20.09 * *$ & 3.62 \\
\hline $\mathbf{P}_{2} \times \mathbf{P}_{9}$ & -1.04 & 5.30 & 5.19 & 4.49 & 3.10 & 4.75 & 1.03 & $37.76^{* *}$ & $-42.16^{* * *}$ & $-8.85^{*}$ & $-22.42 * *$ & $14.14 *$ \\
\hline $\mathbf{P}_{2} \times \mathbf{P}_{10}$ & -1.18 & 5.15 & 3.90 & 3.21 & 2.20 & 3.83 & $39.48^{* * *}$ & $89.23 * *$ & 0.82 & 0.00 & $22.90 * *$ & $44.02 * *$ \\
\hline $\mathbf{P}_{3} \times \mathbf{P}_{4}$ & $5.79^{*}$ & $9.79^{* * *}$ & -4.18 & -0.53 & -1.86 & 2.78 & $43.40^{* *}$ & $41.40^{* *}$ & $42.62^{* *}$ & $13.21^{* *}$ & $45.54^{* *}$ & $27.11^{* *}$ \\
\hline $\mathbf{P}_{3} \times \mathbf{P}_{5}$ & 2.45 & 2.45 & 3.71 & 3.71 & 3.31 & 3.31 & -0.35 & -0.35 & 5.17 & 5.17 & 2.45 & 2.45 \\
\hline $\mathbf{P}_{3} \times \mathbf{P}_{6}$ & 2.97 & -0.48 & $9.52 * *$ & 4.32 & $7.41^{*}$ & 2.78 & $-13.31^{*}$ & $32.52^{* * *}$ & 7.15 & $-8.17^{*}$ & -5.83 & 11.90 \\
\hline $\mathbf{P}_{3} \times \mathbf{P}_{7}$ & 2.18 & $9.52^{* * *}$ & -2.13 & 1.60 & -0.72 & 4.14 & $-38.87 * *$ & 5.59 & $27.38 * *$ & $-8.17^{*}$ & -6.84 & -1.38 \\
\hline $\mathbf{P}_{3} \times \mathbf{P}_{8}$ & -0.52 & $6.50^{*}$ & 3.29 & 3.21 & 2.01 & 4.26 & $-16.82 * *$ & $27.97 * *$ & $-10.26^{* *}$ & -4.77 & $-14.10^{* * *}$ & 11.38 \\
\hline $\mathbf{P}_{3} \times \mathbf{P}_{9}$ & $-7.35^{* * *}$ & -0.69 & 0.19 & 4.01 & -2.28 & 2.50 & $38.46^{* * *}$ & $88.81^{* *}$ & $-37.58^{* * *}$ & -1.63 & -2.81 & $42.98^{* * *}$ \\
\hline $\mathbf{P}_{3} \times \mathbf{P}_{10}$ & $-9.84 * *$ & -3.37 & -0.57 & 3.22 & -3.60 & 1.11 & 6.70 & $44.76^{* *}$ & $-25.26^{* *}$ & $-25.87^{* *}$ & -7.01 & 8.97 \\
\hline $\mathbf{P}_{4} \times \mathbf{P}_{5}$ & -2.31 & -2.31 & -0.71 & -0.71 & -1.22 & -1.22 & $40.91 * *$ & $40.91^{* *}$ & $-25.46^{* * *}$ & $-25.46^{* * *}$ & 7.28 & 7.28 \\
\hline $\mathbf{P}_{4} \times \mathbf{P}_{6}$ & $5.90^{*}$ & 2.35 & $7.26^{* * *}$ & 2.16 & $6.82^{*}$ & 2.22 & $-12.49^{*}$ & $33.78^{* *}$ & $-13.26^{* *}$ & $-25.66^{* *}$ & $-12.77^{*}$ & 3.66 \\
\hline $\mathbf{P}_{4} \times \mathbf{P}_{7}$ & 2.26 & $6.13 *$ & $-6.53 * *$ & -2.53 & -4.28 & 0.24 & $-16.03 * *$ & $45.03 * *$ & $\begin{array}{c}.80 \\
\end{array}$ & $-14.36^{* * *}$ & 8.57 & $14.94 *$ \\
\hline $\mathbf{P}_{4} \times \mathbf{P}_{8}$ & 4.79 & $8.76^{* * *}$ & 1.52 & 1.43 & 1.54 & 3.78 & -1.27 & $51.89 * *$ & 1.15 & 7.35 & -0.27 & $29.32 * *$ \\
\hline $\mathbf{P}_{4} \times \mathbf{P}_{9}$ & -4.97 & -1.37 & -4.49 & 0.44 & -4.64 & -0.14 & $-20.21 * *$ & 8.81 & $-36.54 * *$ & 0.00 & -29.07 *** & 4.35 \\
\hline $\mathbf{P}_{4} \times \mathbf{P}_{10}$ & 3.70 & $7.63^{* * *}$ & -1.71 & 3.37 & 0.01 & 4.73 & 3.56 & $40.49 * *$ & $-44.47 * *$ & $-44.93 * *$ & $-17.04 * *$ & -2.79 \\
\hline $\mathbf{P}_{5} \times \mathbf{P}_{6}$ & 4.28 & 0.79 & 4.71 & -0.27 & 4.57 & 0.07 & $-21.13 * *$ & $20.56^{*}$ & -8.37 & -8.37 & -10.89 & 5.90 \\
\hline $\mathbf{P}_{5} \times \mathbf{P}_{7}$ & $15.14 * *$ & $15.14 * *$ & -1.61 & -1.61 & 3.75 & 3.75 & 0.00 & $72.73 * *$ & $-42.61 * *$ & $-42.61 * *$ & 7.95 & $14.28^{*}$ \\
\hline $\mathbf{P}_{5} \times \mathbf{P}_{8}$ & -1.84 & -1.84 & 3.28 & 3.19 & 1.58 & 1.58 & $25.91 * *$ & $93.71 * *$ & $-30.02^{* *}$ & $-25.73^{* * *}$ & 2.71 & $33.18^{* * *}$ \\
\hline $\mathbf{P}_{5} \times \mathbf{P}_{8}$ & 1.28 & 1.28 & 5.07 & 5.07 & 3.86 & 3.86 & -9.28 & $23.71^{* *}$ & $-27.56^{* * *}$ & $14.16^{* *}$ & $-19.20^{* * *}$ & $18.87^{* * *}$ \\
\hline $\mathbf{P}_{5} \times \mathbf{P}_{10}$ & 4.77 & 4.77 & -0.41 & -0.41 & 1.25 & 1.25 & $31.96 * *$ & $79.02 * *$ & $-14.30^{* *}$ & $-14.30^{* * *}$ & $12.42^{* * *}$ & $31.74 * *$ \\
\hline$\frac{\mathbf{P}_{6} \times \mathbf{P}_{7}}{2}$ & $9.12^{*}$ & $5.46^{*}$ & $9.10^{* * *}$ & 3.92 & $9.11^{1 * *}$ & 4.41 & $-14.98^{* *}$ & $46.85^{* *}$ & $51.63^{* *}$ & $29.95^{* *}$ & $16.37^{* * *}$ & 38.29 ** \\
\hline $\mathbf{P}_{6} \times \mathbf{P}_{8}$ & 4.79 & 1.28 & 4.25 & -0.71 & 4.42 & -0.07 & $12.50^{*}$ & $73.08^{* *}$ & $22.13^{* *}$ & $29.61^{* *}$ & $16.49^{* *}$ & $51.05^{* * *}$ \\
\hline $\mathbf{P}_{6} \times \mathbf{P}_{9}$ & -0.38 & $\begin{array}{l}-3.20 \\
-3.72\end{array}$ & 10.68 *** & $5.41 *$ & $7.10^{*}$ & 2.49 & -9.88 & $37.76^{* *}$ & $-40.52^{* * *}$ & -6.26 & $\frac{1.452^{* *}}{-2}$ & $15.45^{* * *}$ \\
\hline $\mathbf{P}_{6} \times \mathbf{P}_{10}$ & $7.00 * *$ & 3.42 & $7.81^{* *}$ & 2.68 & $7.55^{* * *}$ & 2.92 & $17.43^{*}$ & $79.51 * *$ & $-12.70 * *$ & $-13.41 * *$ & 11.44 & $32.42 * *$ \\
\hline $\mathbf{P}_{7} \times \mathbf{P}_{8}$ & -0.22 & $6.83^{*}$ & -2.42 & -2.51 & -1.69 & 0.48 & -3.28 & $67.06 * *$ & $-15.39^{* * *}$ & $-10.21^{*}$ & -1.36 & $27.91^{* * *}$ \\
\hline $\mathbf{P}_{7} \times \mathbf{P}_{9}$ & -0.82 & $6.50^{*}$ & -0.52 & 3.73 & -0.71 & 4.61 & $-12.23 *$ & $51.61 \mathrm{v}$ & $-40.48^{* *}$ & -6.19 & $-16.86^{* * *}$ & $22.32^{* * *}$ \\
\hline $\mathbf{P}_{7} \times \mathbf{P}_{10}$ & 1.70 & $9.52 *$ & -1.61 & 2.59 & -0.53 & 4.81 & -0.77 & $71.40 * *$ & $-27.32^{* * *}$ & -27.91 ** & 3.33 & $21.08^{* * *}$ \\
\hline $\mathbf{P}_{8} \times \mathbf{P}_{9}$ & -0.88 & 6.12 & 2.23 & 2.14 & 1.19 & 3.42 & 0.82 & $55.10 * *$ & $-14.38^{* * *}$ & $34.92 * *$ & -1.52 & $44.88^{* * *}$ \\
\hline $\mathbf{P}_{8} \times \mathbf{P}_{10}$ & -1.78 & $5.16^{*}$ & 0.72 & 0.63 & -0.12 & 2.08 & $16.86^{* * *}$ & $79.79 * *$ & $10.26^{* *}$ & $17.02^{* *}$ & $14.13^{* * *}$ & $47.98^{* * *}$ \\
\hline $\mathbf{P}_{9} \times \mathbf{P}_{10}$ & 0.48 & $7.90^{*}$ & -0.84 & 4.57 & -0.59 & $5.63 *$ & $-16.92 *$ & 13.29 & $-40.95 * *$ & -6.94 & $-29.96^{* *}$ & 3.04 \\
\hline $\begin{array}{c}\text { No. of crosses with } \\
\text { significant }(+) \text { heterosis }\end{array}$ & 8 & 21 & 6 & 1 & 6 & 2 & 13 & 37 & 8 & 5 & 9 & 25 \\
\hline $\begin{array}{l}\text { No. of crosses with } \\
\text { significant (-) 'heterosis }\end{array}$ & 4 & 0 & 1 & 1 & 0 & 1 & 12 & 0 & 28 & 27 & 13 & 0 \\
\hline Range of heterosis & -9.84 to 15.14 & -3.72 to 18.56 & -6.53 to 10.68 & -7.04 to 5.41 & -4.68 to 9.11 & -5.50 to 7.62 & -38.87 to 75.18 & -0.35 to 93.71 & -68.94 to 51.63 & -61.27 to 34.92 & -29.96 to 45.54 & -12.42 to 51.05 \\
\hline
\end{tabular}

*, ** Significant at 5 per cent and 1 per cent probability levels, respectively. 
Table.1 Cont.....

\begin{tabular}{|c|c|c|c|c|c|c|c|c|c|c|c|c|}
\hline \multirow{3}{*}{ Crosses } & \multicolumn{6}{|c|}{ Number of primary branches per plant } & \multicolumn{6}{|c|}{ Fruit length $(\mathrm{cm})$} \\
\hline & \multicolumn{2}{|c|}{$\mathbf{E}_{1}$} & \multicolumn{2}{|c|}{$\mathbf{E}_{2}$} & \multicolumn{2}{|c|}{ Pooled } & \multicolumn{2}{|c|}{$E_{1}$} & \multicolumn{2}{|c|}{$\mathbf{E}_{2}$} & \multicolumn{2}{|c|}{ Pooled } \\
\hline & $\mathbf{B P}$ & SV & BP & SV & BP & SV & $\mathbf{B P}$ & SV & $\mathbf{B P}$ & SV & $\mathbf{B P}$ & SV \\
\hline$\overline{\mathbf{P}_{1} \times \mathbf{P}_{2}}$ & -1.23 & 9.20 & $-44.65 * *$ & $-22.03 *$ & $-17.31^{* * *}$ & -0.67 & $-18.49 * *$ & -1.17 & $-17.08^{* * *}$ & 2.86 & $-17.83^{* *}$ & 0.68 \\
\hline $\mathbf{P}_{1} \times \mathbf{P}_{3}$ & $-29.82 * *$ & $-15.50^{*}$ & $18.70^{*}$ & $39.02 * *$ & $-14.78^{*}$ & 1.71 & -4.92 & $20.79 * *$ & -5.74 & $23.00 * *$ & -5.30 & $21.81 * *$ \\
\hline $\mathbf{P}_{1} \times \mathbf{P}_{4}$ & 4.92 & $-20.81 * *$ & -11.12 & $37.74^{* *}$ & -2.89 & -2.32 & $-14.40^{* * *}$ & -6.32 & -0.71 & 4.10 & -1.28 & -1.53 \\
\hline $\mathbf{P}_{1} \times \mathbf{P}_{5}$ & -9.52 & -9.52 & 2.90 & 6.64 & -4.42 & -4.42 & -6.03 & -6.03 & $-16.45^{* * *}$ & $-12.40 * *$ & $-8.97 *$ & $-8.97 *$ \\
\hline $\mathbf{P}_{1} \times \mathbf{P}_{6}$ & $31.21^{* *}$ & $33.07 * *$ & $25.88^{* *}$ & $74.27 * *$ & $29.15^{* * *}$ & $46.08^{* *}$ & 5.56 & $-24.07 * *$ & $-25.67^{* * *}$ & $-22.07^{* * *}$ & $-11.76^{*}$ & $-23.15^{* * *}$ \\
\hline $\mathbf{P}_{1} \times \mathbf{P}_{7}$ & -4.70 & 6.28 & $-32.61 * *$ & 16.28 & $-16.33^{* * *}$ & 9.44 & -13.67 ** & -15.11 ** & $-12.43^{* * *}$ & -0.60 & $-13.06^{* * *}$ & $-8.42^{*}$ \\
\hline $\mathbf{P}_{1} \times \mathbf{P}_{8}$ & 2.68 & $27.41^{* *}$ & $64.24 * *$ & $94.76^{* * *}$ & $21.53 * *$ & $48.68 * *$ & $-25.58 * *$ & -4.59 & 4.49 & $21.85^{* *}$ & $-12.44 * *$ & $7.59 *$ \\
\hline $\mathbf{P}_{1} \times \mathbf{P}_{9}$ & 9.33 & $27.73^{* * *}$ & $39.62^{* * *}$ & $44.70^{* * *}$ & $22.95 * *$ & $33.09 * *$ & $-8.22^{*}$ & 4.69 & 1.34 & $18.53^{* * *}$ & -3.75 & $11.06^{* *}$ \\
\hline $\mathbf{P}_{1} \times \mathbf{P}_{10}$ & $-23.22^{* * *}$ & $12.29 *$ & $72.89^{* * *}$ & $79.18^{* * *}$ & 0.84 & $33.41^{* * *}$ & $-11.55 * *$ & 1.15 & $-17.37 * *$ & -0.16 & $-14.31^{* * *}$ & 0.54 \\
\hline $\mathbf{P}_{2} \times \mathbf{P}_{3}$ & -5.39 & $13.91 *$ & -28.01 *** & 1.40 & -8.46 & 9.96 & 3.56 & $31.57^{* *}$ & $-26.17^{* * *}$ & -3.65 & $-10.33^{* * *}$ & $15.35^{* * *}$ \\
\hline $\mathbf{P}_{2} \times \mathbf{P}_{4}$ & $15.38^{* *}$ & $27.56^{* *}$ & $-30.57 * *$ & 7.60 & 0.94 & $21.25^{* *}$ & -0.63 & $20.49 * *$ & $9.40 * *$ & $35.69 * *$ & 4.05 & $27.49 * *$ \\
\hline $\mathbf{P}_{2} \times \mathbf{P}_{5}$ & -0.75 & 9.73 & 4.35 & $47.00^{* * *}$ & 1.14 & $21.50^{* * *}$ & -2.95 & $17.67 * *$ & $-8.93^{* *}$ & $12.95^{* *}$ & -5.74 & $15.50^{* * *}$ \\
\hline $\mathbf{P}_{2} \times \mathbf{P}_{6}$ & $-27.35 * *$ & $-19.69 * *$ & -11.20 & $25.10^{* *}$ & $-21.37^{* * *}$ & -5.55 & $-14.41 * *$ & 3.78 & $-7.58^{*}$ & $14.63^{* *}$ & $-11.23^{* *}$ & 8.77 \\
\hline $\mathbf{P}_{2} \times \mathbf{P}_{7}$ & $-31.74 * *$ & $-23.87 * *$ & $-47.41 * *$ & -9.26 & $-38.27^{* * *}$ & $-19.26^{* * *}$ & 0.11 & $21.38^{* * *}$ & 2.19 & $26.75^{* *}$ & 1.08 & $23.85^{* * *}$ \\
\hline $\mathbf{P}_{2} \times \mathbf{P}_{8}$ & 0.02 & $24.11^{* *}$ & $17.36^{* * *}$ & $65.33^{* *}$ & $12.08^{*}$ & $37.12 * *$ & $-14.36^{* * *}$ & $9.80^{*}$ & -3.73 & $19.41^{* *}$ & $-7.04 *$ & $14.23 * *$ \\
\hline $\mathbf{P}_{2} \times \mathbf{P}_{9}$ & -5.70 & 10.17 & $-17.45^{* * *}$ & 16.28 & -6.68 & 12.10 & $-9.72^{* * *}$ & $9.46^{*}$ & $-11.31^{\text {*** }}$ & $10.00^{*}$ & $-10.46^{* * *}$ & $9.71^{*}$ \\
\hline $\mathbf{P}_{2} \times \mathbf{P}_{10}$ & $-14.09^{* * *}$ & $25.64 * *$ & $13.28^{*}$ & $59.58^{* * *}$ & 3.06 & $36.36^{* * *}$ & -2.96 & $17.66^{* * *}$ & 3.77 & $28.72^{* * *}$ & 0.18 & $22.75^{* * *}$ \\
\hline $\mathbf{P}_{3} \times \mathbf{P}_{4}$ & -8.67 & 9.96 & $-37.33^{* *}$ & -2.87 & -11.27 & 5.91 & $-7.65^{*}$ & $17.32^{* * *}$ & $-6.28^{*}$ & $22.30^{* *}$ & $-7.01^{*}$ & $19.62^{* * *}$ \\
\hline $\mathbf{P}_{3} \times \mathbf{P}_{5}$ & -3.84 & $15.77^{* * *}$ & $25.90^{* * *}$ & $47.45^{* * *}$ & 5.7 & $25.77^{* * *}$ & $-9.60^{* * *}$ & $14.85^{* *}$ & $-9.54 * *$ & $18.04 * *$ & $-9.57^{* * *}$ & $16.32^{* *}$ \\
\hline $\mathbf{P}_{3} \times \mathbf{P}_{6}$ & $-18.16^{* * *}$ & -1.47 & $29.20^{* * *}$ & $78.86^{* * *}$ & 3.80 & $23.90^{* * *}$ & $-26.55 * *$ & -6.68 & $-14.26^{* *}$ & $11.89^{* * *}$ & $-20.81 * *$ & 1.87 \\
\hline $\mathbf{P}_{3} \times \mathbf{P}_{7}$ & $-11.29^{*}$ & 6.81 & $-30.83 * *$ & $19.35^{*}$ & $-15.31^{* * *}$ & 10.77 & $-15.78 * *$ & 7.00 & -3.29 & $26.20 * *$ & $-9.94 * *$ & $15.84 * *$ \\
\hline $\mathbf{P}_{3} \times \mathbf{P}_{8}$ & -6.32 & $16.24 * *$ & $-15.46^{*}$ & 0.26 & -9.11 & 11.19 & $-20.69 * *$ & 1.69 & $-9.02 * *$ & $18.72^{* *}$ & $-14.85^{* *}$ & $9.53^{*}$ \\
\hline $\mathbf{P}_{3} \times \mathbf{P}_{9}$ & $-16.25 * *$ & 0.83 & $46.51^{* * *}$ & $71.58^{* * *}$ & 3.19 & $23.17 * *$ & -3.85 & $22.15 * *$ & $-13.50 * *$ & $12.87^{* * *}$ & $-8.36 * *$ & $17.88^{* * *}$ \\
\hline $\mathbf{P}_{3} \times \mathbf{P}_{10}$ & $-12.27 * *$ & $28.29^{* * *}$ & $-17.07^{*}$ & -2.87 & -10.47 & $18.45^{* * *}$ & -2.34 & $24.08^{* *}$ & -5.94 & $22.75^{* *}$ & -4.02 & $23.47 * *$ \\
\hline $\mathbf{P}_{4} \times \mathbf{P}_{5}$ & 9.87 & 9.87 & -8.28 & $42.15^{* * *}$ & $19.37 * *$ & $20.06^{* * *}$ & $-12.10^{* *}$ & -3.81 & -4.70 & -4.70 & -4.22 & -4.22 \\
\hline $\mathbf{P}_{4} \times \mathbf{P}_{6}$ & 1.92 & 3.36 & 7.13 & $66.03^{* *}$ & 8.88 & $23.15 * *$ & $-21.83^{* *}$ & $-14.46^{* *}$ & $21.70^{* * *}$ & $14.38^{* * *}$ & -0.93 & -1.17 \\
\hline $\mathbf{P}_{4} \times \mathbf{P}_{7}$ & $-36.31 * *$ & $-28.97 * *$ & $-12.25^{*}$ & $51.40^{* * *}$ & $-26.29 * *$ & -3.59 & $9.65^{*}$ & $19.99 * *$ & $-14.45^{* * *}$ & -2.89 & 3.91 & $9.45^{*}$ \\
\hline $\mathbf{P}_{4} \times \mathbf{P}_{8}$ & $-10.36^{*}$ & 11.23 & $-18.09 * *$ & $26.95^{* *}$ & -5.03 & $16.19 *$ & -5.93 & $20.61 * *$ & $-13.04 * *$ & 1.40 & $-9.04 * *$ & $11.77^{* * *}$ \\
\hline $\mathbf{P}_{4} \times \mathbf{P}_{9}$ & 8.32 & $26.55^{* * *}$ & $-30.57 * *$ & 7.60 & 11.38 & $20.57 * *$ & $12.55^{* * *}$ & $28.37 * *$ & $24.99^{* *}$ & $46.20 * *$ & $18.36^{* *}$ & $36.58^{* * *}$ \\
\hline $\mathbf{P}_{4} \times \mathbf{P}_{10}$ & $-12.23 * *$ & $28.35^{* * *}$ & $12.81 *$ & $74.84^{* * *}$ & 8.11 & $43.03 * *$ & 0.17 & $14.56^{* *}$ & 5.31 & $27.23^{* *}$ & 2.61 & $20.39 * *$ \\
\hline $\mathbf{P}_{5} \times \mathbf{P}_{6}$ & 6.04 & 7.54 & $17.53^{* * *}$ & $62.71^{* *}$ & 10.48 & $24.96 * *$ & $-12.13^{* *}$ & $-12.13^{* *}$ & -2.22 & -2.22 & $-7.57^{*}$ & $-7.57^{*}$ \\
\hline $\mathbf{P}_{5} \times \mathbf{P}_{7}$ & $-35.23 * *$ & $-27.76^{* *}$ & $-42.23^{* * *}$ & -0.32 & $-38.14^{* * *}$ & $-19.10^{* * *}$ & -4.72 & -4.72 & $-9.80^{* * *}$ & 2.39 & -6.43 & -1.44 \\
\hline $\mathbf{P}_{5} \times \mathbf{P}_{8}$ & 9.62 & $36.02 * *$ & $64.73^{* * *}$ & $95.34^{* * *}$ & $26.49^{* * *}$ & $54.75^{* * *}$ & -3.28 & $24.01^{* * *}$ & 2.83 & $19.92^{* *}$ & -0.61 & 22.12 \\
\hline $\mathbf{P}_{5} \times \mathbf{P}_{9}$ & 2.67 & $19.95^{* * *}$ & $39.21^{* * *}$ & $39.21^{* *}$ & $16.43^{*}$ & $26.03 * *$ & -4.12 & $9.36^{*}$ & $-8.72 *$ & 6.76 & -6.27 & $8.16^{*}$ \\
\hline $\mathbf{P}_{5} \times \mathbf{P}_{10}$ & $-39.10^{* * *}$ & -10.93 & $29.77^{* * *}$ & $32.50^{* *}$ & $-22.31^{* *}$ & 2.78 & -6.10 & 7.38 & $-9.54 * *$ & $9.29 *$ & $-7.73^{*}$ & $8.26^{*}$ \\
\hline $\mathbf{P}_{6} \times \mathbf{P}_{7}$ & $-30.71 * *$ & $-22.72 * *$ & $-16.84^{* * *}$ & $43.49^{* *}$ & $-24.93^{* *}$ & -1.81 & $36.37^{* * *}$ & $34.11 * *$ & $-9.62^{* * *}$ & 2.60 & $13.54 * *$ & $19.60^{* * *}$ \\
\hline $\mathbf{P}_{6} \times \mathbf{P}_{8}$ & $9.86^{*}$ & $36.31^{* * *}$ & $14.81^{*}$ & $58.94^{* *}$ & $17.26^{* *}$ & $43.46^{* * *}$ & $-22.29 * *$ & -0.37 & -6.61 & $8.90^{*}$ & $-15.44^{* * *}$ & 3.90 \\
\hline $\mathbf{P}_{6} \times \mathbf{P}_{9}$ & $-27.85 * *$ & $-15.71^{*}$ & $19.42^{* * *}$ & $65.33^{* * *}$ & -2.85 & 9.88 & $-34.95 * *$ & $-25.81 * *$ & $-15.01 * *$ & -0.59 & $-25.64 * *$ & $-14.19 * *$ \\
\hline $\mathbf{P}_{6} \times \mathbf{P}_{10}$ & -7.80 & $34.84 * *$ & $15.13^{*}$ & $59.39^{* *}$ & 7.77 & $42.59 * *$ & $-27.78^{* * *}$ & $-17.41 * *$ & $-11.50^{* * *}$ & 6.93 & $-20.06^{* * *}$ & -6.20 \\
\hline $\mathbf{P}_{7} \times \mathbf{P}_{8}$ & 2.99 & $27.79 * *$ & $-17.51 * *$ & $42.34 * *$ & 1.22 & 32.39 & $-14.94 * *$ & $9.06^{*}$ & 0.52 & $17.22 * *$ & $-8.18^{*}$ & $12.82 * *$ \\
\hline $\mathbf{P}_{7} \times \mathbf{P}_{9}$ & $13.40^{*}$ & $32.48 * *$ & -6.14 & $61.94 * *$ & 8.40 & $41.78 * *$ & $-9.13^{*}$ & 3.65 & $-15.22^{* * *}$ & -0.83 & $-11.97^{* *}$ & 1.58 \\
\hline $\mathbf{P}_{7} \times \mathbf{P}_{10}$ & 0.95 & $47.63 * *$ & $-49.74 * *$ & -13.28 & -2.96 & $28.39^{* * *}$ & -4.07 & $9.70^{*}$ & -3.86 & $16.15^{* * *}$ & -3.97 & $12.67^{* * *}$ \\
\hline$P_{8} \times P 9$ & 8.22 & 34.28 *** & $50.73^{* * *}$ & $78.74^{* * *}$ & $21.23 * *$ & $48.32 * *$ & $-12.96^{* * *}$ & $11.60 * *$ & -0.69 & $16.16^{* *}$ & $-7.47^{*}$ & $13.70^{* * *}$ \\
\hline $\mathbf{P}_{8} \times \mathbf{P}_{10}$ & -3.87 & $40.58^{* * *}$ & $35.92^{* * *}$ & $61.17^{* *}$ & $11.17^{*}$ & $47.09^{* *}$ & -6.06 & $20.44 * *$ & 0.11 & $20.95^{* *}$ & -1.79 & $20.67^{* * *}$ \\
\hline $\mathbf{P}_{9} \times \mathbf{P}_{10}$ & $-25.43^{* *}$ & 9.05 & $73.80^{* * *}$ & $77.46^{* *}$ & -1.25 & $30.65 * *$ & $-28.47 * *$ & $-18.20^{* *}$ & -1.41 & $19.11^{* *}$ & $-15.64 * *$ & -1.02 \\
\hline $\begin{array}{l}\text { No. of crosses with } \\
\text { significant }(+) \text { heterosis }\end{array}$ & 4 & 22 & 21 & 32 & 10 & 27 & 3 & 23 & 3 & 28 & 2 & 27 \\
\hline $\begin{array}{l}\text { No. of crosses with } \\
\text { significant (-) 'heterosis }\end{array}$ & 17 & 8 & 17 & 1 & 10 & 2 & 23 & 7 & 23 & 2 & 25 & 5 \\
\hline Range of heterosis & -39.10 to 31.21 & -28.97 to 47.63 & -49.74 to 73.80 & -22.03 to 95.34 & $\begin{array}{l}-38.27 \text { to } \\
29.15\end{array}$ & -19.26 to 54.75 & -34.95 to 36.37 & -25.81 to 34.11 & -26.17 to 24.99 & -22.07 to 46.20 & -25.64 to 18.36 & -23.15 to 36.58 \\
\hline
\end{tabular}

$*, * *$ Significant at 5 per cent and 1 per cent probability levels, respectively. 
Table.1 Cont.....

\begin{tabular}{|c|c|c|c|c|c|c|c|c|c|c|c|c|}
\hline \multirow{3}{*}{ Crosses } & \multicolumn{6}{|c|}{ Fruit circumference $(\mathrm{cm})$} & \multicolumn{6}{|c|}{ Fruit weight (kg) } \\
\hline & \multicolumn{2}{|c|}{$E_{1}$} & \multicolumn{2}{|c|}{$\mathbf{E}_{2}$} & \multicolumn{2}{|c|}{ Pooled } & \multicolumn{2}{|c|}{$E_{1}$} & \multicolumn{2}{|c|}{$\mathbf{E}_{2}$} & \multicolumn{2}{|c|}{ Pooled } \\
\hline & $\mathbf{B P}$ & SV & BP & SV & BP & SV & $\mathbf{B P}$ & SV & $\mathbf{B P}$ & SV & $\mathbf{B P}$ & SV \\
\hline $\mathbf{P}_{1} \times \mathbf{P}_{2}$ & $-20.41^{* *}$ & $10.51^{*}$ & $-30.47 * *$ & $11.28^{* * *}$ & $-25.87 * *$ & $10.90^{*}$ & -6.91 & 3.33 & $-18.79 * *$ & 7.63 & -12.82 & 5.28 \\
\hline $\mathbf{P}_{1} \times \mathbf{P}_{3}$ & $-27.07 * *$ & 1.26 & $-11.04^{* * *}$ & $42.39 * *$ & $-18.38^{* * *}$ & $22.10^{* *}$ & $-22.52^{* *}$ & -14.00 & -11.52 & 17.27 & $-17.04 *$ & 0.18 \\
\hline $\mathbf{P}_{1} \times \mathbf{P}_{4}$ & $-9.59 * *$ & $25.53^{* * *}$ & $-30.47^{* * *}$ & $11.30^{* *}$ & $-20.91 * *$ & $18.31 * *$ & -14.41 & -5.00 & $-14.24 *$ & 13.65 & $-14.33^{*}$ & 3.46 \\
\hline $\mathbf{P}_{1} \times \mathbf{P}_{5}$ & $-8.86^{*}$ & $26.55^{\text {*** }}$ & $-37.24 * *$ & 0.46 & $-24.25^{* * *}$ & $13.33^{* *}$ & $-21.02^{* * *}$ & -12.33 & $-30.91^{* * *}$ & -8.43 & $-25.94 * *$ & -10.56 \\
\hline $\mathbf{P}_{1} \times \mathbf{P}_{6}$ & $8.01 * *$ & $94.25 * *$ & $-35.08 * *$ & $8.68^{*}$ & $-13.06 * *$ & $50.88 * *$ & $-17.12 *$ & -8.00 & $-22.98 * *$ & $22.49 *$ & -12.37 & 5.83 \\
\hline $\mathbf{P}_{1} \times \mathbf{P}_{7}$ & $-10.97 * *$ & $23.61^{* *}$ & $-33.22^{* * *}$ & 6.89 & $-23.03 * *$ & $15.14 * *$ & -8.71 & 1.33 & $-48.68 * *$ & -6.02 & $-29.21^{* *}$ & -2.00 \\
\hline $\mathbf{P}_{1} \times \mathbf{P}_{8}$ & $-20.43 * *$ & $10.48^{*}$ & $-22.83 * *$ & $23.51 * *$ & $-21.73 * *$ & $17.08 * *$ & -8.41 & 1.67 & 5.15 & $39.36^{* * *}$ & -1.66 & $18.76^{*}$ \\
\hline $\mathbf{P}_{1} \times \mathbf{P}_{9}$ & $-26.68 * *$ & 1.81 & $-20.91 * *$ & $26.58^{* * *}$ & $-23.55^{* *}$ & $14.36^{* * *}$ & $-18.02^{*}$ & -9.00 & $-17.58^{*}$ & 9.24 & $-17.80^{*}$ & -0.73 \\
\hline $\mathbf{P}_{1} \times \mathbf{P}_{10}$ & -6.66 & $29.59 * *$ & $-38.44 * *$ & -1.47 & $-23.89 * *$ & $13.85^{* *}$ & -4.20 & 6.33 & -3.18 & $59.04 * *$ & 5.77 & $30.24 * *$ \\
\hline $\mathbf{P}_{2} \times \mathbf{P}_{3}$ & 7.15 & $11.64^{*}$ & $-15.39^{* * *}$ & -1.31 & -0.02 & 5.07 & $42.49 * *$ & $48.67 * *$ & -14.48 & -0.40 & $16.44 *$ & $26.41^{* * *}$ \\
\hline $\mathbf{P}_{2} \times \mathbf{P}_{4}$ & -7.63 & 1.71 & $18.82^{* * *}$ & $15.30^{* * *}$ & 4.94 & 8.60 & -14.51 & -9.67 & $17.24 *$ & $36.55^{* *}$ & 1.50 & 11.29 \\
\hline $\mathbf{P}_{2} \times \mathbf{P}_{5}$ & 1.16 & 5.40 & -7.43 & -7.43 & -1.10 & -1.10 & $-26.80^{* *}$ & $-25.33^{* *}$ & 10.69 & $28.92 * *$ & -8.56 & -0.73 \\
\hline $\mathbf{P}_{2} \times \mathbf{P}_{6}$ & $-45.48 * *$ & -1.95 & $-25.81^{* *}$ & $24.20^{* * *}$ & $-35.86 * *$ & $11.30 *$ & -7.19 & -5.33 & $-36.11 * *$ & 1.61 & $-15.83^{*}$ & -2.19 \\
\hline $\mathbf{P}_{2} \times \mathbf{P}_{7}$ & $10.18^{*}$ & $14.80^{* * *}$ & $-7.15^{*}$ & $20.61^{* *}$ & 2.52 & $17.74 * *$ & $-18.63^{*}$ & $-17.00^{*}$ & $-52.63^{* * *}$ & -13.25 & $-38.82^{* * *}$ & -15.30 \\
\hline $\mathbf{P}_{2} \times \mathbf{P}_{8}$ & 8.40 & $15.28^{* * *}$ & -4.84 & -5.43 & 1.91 & 4.79 & -8.17 & -6.33 & $16.90^{*}$ & $36.14 * *$ & 4.03 & 12.93 \\
\hline $\mathbf{P}_{2} \times \mathbf{P}_{9}$ & -3.30 & 0.75 & $19.16^{* * *}$ & 5.72 & 7.60 & 3.27 & -14.05 & -12.33 & $15.86^{*}$ & $34.94^{* * *}$ & 0.50 & 9.11 \\
\hline $\mathbf{P}_{2} \times \mathbf{P}_{10}$ & -3.32 & 4.19 & $10.45^{*}$ & 7.14 & 3.29 & 5.69 & $-16.01 *$ & -14.33 & $-33.25^{* * *}$ & 9.64 & $-21.60^{* * *}$ & -3.46 \\
\hline $\mathbf{P}_{3} \times \mathbf{P}_{4}$ & $-9.32^{*}$ & -0.14 & $-22.83^{* * *}$ & $-10.00^{*}$ & $-9.74 *$ & -5.14 & -15.14 & -10.33 & -14.74 & -2.41 & $-14.95^{*}$ & -6.74 \\
\hline $\mathbf{P}_{3} \times \mathbf{P}_{5}$ & 1.92 & 1.92 & -6.58 & $8.96^{*}$ & 0.37 & 5.48 & $-26.84 * *$ & $-23.67 * *$ & 12.45 & 12.45 & -9.27 & -7.29 \\
\hline $\mathbf{P}_{3} \times \mathbf{P}_{6}$ & $-35.90^{* * *}$ & $15.28^{* * *}$ & $-28.21 * *$ & $20.18^{* * *}$ & $-32.14 * *$ & $17.76^{* * *}$ & $-22.68 * *$ & $-19.33^{*}$ & $-28.79 * *$ & 13.25 & $-17.87 *$ & -4.55 \\
\hline $\mathbf{P}_{3} \times \mathbf{P}_{7}$ & 8.06 & 7.38 & -4.92 & $23.51^{* * *}$ & 0.62 & $15.56 * *$ & $-30.99 * *$ & $-28.00^{* *}$ & $-45.83 * *$ & -0.80 & $-39.08 * *$ & -15.66 \\
\hline $\mathbf{P}_{3} \times \mathbf{P}_{8}$ & -4.25 & 1.82 & -6.40 & $9.17^{*}$ & 0.43 & 5.55 & -9.90 & -6.00 & 8.06 & 7.63 & \begin{tabular}{|l|}
-1.96 \\
\end{tabular} & 0.18 \\
\hline $\mathbf{P}_{3} \times \mathbf{P}_{9}$ & $23.24 * *$ & $19.97^{* * *}$ & $31.06^{* * *}$ & $52.86^{* *}$ & $30.01^{* * *}$ & $36.64 * *$ & -8.31 & -4.33 & $43.95^{* * *}$ & $43.37 * *$ & $14.80^{*}$ & $17.30^{*}$ \\
\hline $\mathbf{P}_{3} \times \mathbf{P}_{10}$ & 6.92 & $15.24 * *$ & $-26.87 * *$ & $-14.70^{* * *}$ & -4.79 & 0.06 & $-19.17 *$ & -15.67 & $-54.28^{* *}$ & $-24.90^{* * *}$ & $-34.91 * *$ & $-19.85^{*}$ \\
\hline $\mathbf{P}_{4} \times \mathbf{P}_{5}$ & $-13.42^{* *}$ & -4.67 & $43.79^{* *}$ & $43.79 * *$ & $15.86^{* * *}$ & $19.89^{* *}$ & $32.49 * *$ & $40.00 * *$ & $31.58^{* *}$ & $50.60^{* *}$ & $32.06 * *$ & $44.81 * *$ \\
\hline $\mathbf{P}_{4} \times \mathbf{P}_{6}$ & $-17.62^{* * *}$ & $48.16^{* * *}$ & $-32.78^{* * *}$ & $12.53 * *$ & $-25.03 * *$ & $30.11 * *$ & -11.36 & -6.33 & $-27.02 * *$ & 16.06 & -10.66 & 3.83 \\
\hline $\mathbf{P}_{4} \times \mathbf{P}_{7}$ & $-9.71 *$ & -0.58 & $-12.00^{* * *}$ & $14.31^{* *}$ & -6.86 & 6.96 & -11.67 & -6.67 & $-50.22 * *$ & -8.84 & $-33.29^{* * *}$ & -7.65 \\
\hline $\mathbf{P}_{4} \times \mathbf{P}_{8}$ & 5.16 & $15.80 * *$ & $11.67^{* * *}$ & $10.99 *$ & 9.54* & $13.36^{* * *}$ & -11.36 & -6.33 & $19.65^{*}$ & $36.95^{* * *}$ & 3.32 & 13.30 \\
\hline $\mathbf{P}_{4} \times \mathbf{P}_{9}$ & $11.44^{* * *}$ & $22.71^{* * *}$ & $18.16^{* * *}$ & $14.66^{* * *}$ & $14.64^{* * *}$ & $18.63^{* *}$ & -0.32 & 5.33 & 3.16 & 18.07 & 1.33 & 11.11 \\
\hline $\mathbf{P}_{4} \times \mathbf{P}_{10}$ & $8.79^{*}$ & $19.79 * *$ & $12.32^{* * *}$ & $8.99^{*}$ & $10.47^{*}$ & $14.32 * *$ & -8.20 & -3.00 & -6.60 & $53.41^{\text {*** }}$ & -0.44 & $22.59 * *$ \\
\hline $\mathbf{P}_{5} \times \mathbf{P}_{6}$ & $-17.84^{* *}$ & $47.77^{* * *}$ & $-25.17 * *$ & $25.27 * *$ & $-21.42 * *$ & $36.37 * *$ & $-26.33^{* *}$ & $-26.33^{* *}$ & $-18.94 * *$ & $28.92 * *$ & $-15.05^{*}$ & -1.28 \\
\hline $\mathbf{P}_{5} \times \mathbf{P}_{7}$ & -7.24 & -7.24 & $-11.63^{* *}$ & $14.80^{* * *}$ & $-9.50^{*}$ & 3.93 & $-17.43^{*}$ & $-16.33^{*}$ & $-51.32 * *$ & -10.84 & $-37.76 * *$ & -13.84 \\
\hline $\mathbf{P}_{5} \times \mathbf{P}_{8}$ & -2.58 & 3.60 & $24.27^{* * *}$ & $24.27^{* *}$ & $10.95^{*}$ & $14.08 * *$ & 5.67 & 5.67 & $93.57^{* * *}$ & $93.57^{* *}$ & $45.54 * *$ & $45.54 * *$ \\
\hline $\mathbf{P}_{5} \times \mathbf{P}_{9}$ & 5.12 & 5.12 & -2.78 & -2.78 & 1.12 & 1.12 & 35.00 *** & $35.00^{* * *}$ & $-21.29 *$ & $-21.29^{*}$ & 9.47 & 9.47 \\
\hline $\mathbf{P}_{5} \times \mathbf{P}_{10}$ & -7.65 & -0.47 & $-11.92 * *$ & $-11.92 * *$ & -8.40 & -6.28 & -2.33 & -2.33 & $-49.39 * *$ & -16.87 & $-26.04 * *$ & -8.93 \\
\hline $\mathbf{P}_{6} \times \mathbf{P}_{7}$ & $-37.01 * *$ & $13.29 * *$ & $-9.37 * *$ & $51.73^{* * *}$ & $-23.49 * *$ & $32.77 * *$ & -8.22 & -7.00 & $10.09 *$ & $101.61^{* * *}$ & 2.76 & $42.26 * *$ \\
\hline $\mathbf{P}_{6} \times \mathbf{P}_{8}$ & $-12.31^{* * *}$ & $57.71^{* * *}$ & $-23.32^{* * *}$ & $28.37^{* * *}$ & $-17.69^{* * *}$ & $42.84 * *$ & -5.32 & -11.00 & $-26.01^{* * *}$ & 17.67 & -12.23 & 2.00 \\
\hline $\mathbf{P}_{6} \times \mathbf{P}_{9}$ & $-29.34 * *$ & $27.08 * *$ & $-21.69^{* *}$ & $31.09 * *$ & $-25.60 * *$ & $29.11 * *$ & -4.07 & -13.67 & $-21.46^{* * *}$ & $24.90 * *$ & -10.66 & 3.83 \\
\hline $\mathbf{P}_{6} \times \mathbf{P}_{10}$ & $-12.70^{* * *}$ & $57.01 * *$ & $-27.02 * *$ & $22.18 * *$ & $-19.70^{* * *}$ & $39.36 * *$ & 6.74 & -5.00 & $27.14 * *$ & $108.84 * *$ & $19.08 * *$ & $46.63 * *$ \\
\hline $\mathbf{P}_{7} \times \mathbf{P}_{8}$ & 0.97 & 7.38 & -4.80 & $23.66 * *$ & 0.69 & $15.63 * *$ & $-17.43^{*}$ & $-16.33^{*}$ & $-36.40^{* * *}$ & 16.47 & $-28.82 * *$ & -1.46 \\
\hline $\mathbf{P}_{7} \times \mathbf{P}_{9}$ & 5.75 & 5.09 & 1.32 & $31.61^{* *}$ & 3.21 & $18.53^{* *}$ & -8.88 & -7.67 & $-23.25^{* * *}$ & $40.56^{* * *}$ & $-17.50^{* * *}$ & 14.21 \\
\hline $\mathbf{P}_{7} \times \mathbf{P}_{10}$ & 0.71 & 8.55 & $-11.20^{* * *}$ & 15.35 ** & -2.48 & $11.99 * *$ & -3.62 & -2.33 & $-42.32 * *$ & 5.62 & $-26.84 * *$ & 1.28 \\
\hline $\mathbf{P}_{8} \times \mathbf{P 9}$ & 8.01 & 14.86 *** & 4.74 & 4.10 & 6.40 & $9.41 *$ & -2.84 & -8.67 & $28.22 *$ & 4.02 & 12.92 & -2.91 \\
\hline $\mathbf{P}_{8} \times \mathbf{P}_{10}$ & $15.44 * *$ & $24.41 * *$ & 6.91 & 6.25 & $12.05^{* * *}$ & $15.21 * *$ & 1.42 & -4.67 & $-32.76^{* * *}$ & 10.44 & $-17.01 *$ & 2.19 \\
\hline $\mathbf{P}_{9} \times \mathbf{P}_{10}$ & 3.47 & $11.51^{*}$ & $39.39 * *$ & $35.22 * *$ & $20.73^{* * *}$ & $23.53 * *$ & 12.22 & 1.00 & $-32.03 * *$ & 11.65 & $-14.05 *$ & 5.83 \\
\hline $\begin{array}{c}\text { No. of crosses with } \\
\text { significant }(+) \text { heterosis }\end{array}$ & 6 & 25 & 10 & 31 & 8 & 31 & 3 & 3 & 9 & 17 & 5 & 9 \\
\hline $\begin{array}{c}\text { No. of crosses with } \\
\text { significant (-) 'heterosis }\end{array}$ & 18 & o & 25 & 3 & 19 & $\mathbf{0}$ & 14 & 8 & 25 & 2 & 22 & 1 \\
\hline Range of heterosis & -45.48 to 23.24 & -7.24 to 94.25 & -38.44 to 43.79 & -14.70 to 52.86 & -35.86 to 20.73 & -6.28 to 50.88 & -30.99 to 42.49 & -28.00 to 48.67 & -54.28 to 93.57 & -24.90 to 108.84 & -39.08 to 45.54 & -19.85 to 46.63 \\
\hline
\end{tabular}

*, ** Significant at 5 per cent and 1 per cent probability levels, respectively. 
Table.1 Cont.....

\begin{tabular}{|c|c|c|c|c|c|c|c|c|c|c|c|c|}
\hline \multirow{3}{*}{ Crosses } & \multicolumn{6}{|c|}{ Number of fruits per plant } & \multicolumn{6}{|c|}{ Fruit yield per plant (kg) } \\
\hline & \multicolumn{2}{|c|}{$\mathbf{E}_{1}$} & \multicolumn{2}{|c|}{$\mathbf{E}_{2}$} & \multicolumn{2}{|c|}{ Pooled } & \multicolumn{2}{|c|}{$\mathbf{E}_{1}$} & \multicolumn{2}{|c|}{$\mathrm{E}_{2}$} & \multicolumn{2}{|c|}{ Pooled } \\
\hline & $\mathbf{B P}$ & SV & BP & SV & BP & SV & $\mathbf{B P}$ & SV & $\mathbf{B P}$ & SV & $\mathbf{B P}$ & SV \\
\hline$\overline{\mathbf{P}_{1} \times \mathbf{P}_{2}}$ & $36.11^{* *}$ & 20.01 & $66.93 * *$ & $45.80^{* * *}$ & $50.88^{* * *}$ & $32.44 * *$ & $26.40 * *$ & $24.09 * *$ & $35.42^{* *}$ & $57.06^{* *}$ & $30.70^{* *}$ & $38.45^{* *}$ \\
\hline $\mathbf{P}_{1} \times \mathbf{P}_{3}$ & $70.52^{* * *}$ & $50.36^{* * *}$ & $32.69^{* * *}$ & $20.07 *$ & $53.85^{* * *}$ & $35.77 * *$ & $31.85^{* * *}$ & $29.44 * *$ & $20.70^{* * *}$ & $39.98^{* *}$ & $26.53^{* * *}$ & $34.03 * *$ \\
\hline $\mathbf{P}_{1} \times \mathbf{P}_{4}$ & $40.97 * *$ & $24.30 *$ & $38.19^{* * *}$ & $20.70^{*}$ & $39.64 * *$ & $22.57 *$ & $20.41^{*}$ & $18.21^{*}$ & $17.20^{*}$ & $35.93^{* * *}$ & $18.88^{*}$ & $25.93^{* *}$ \\
\hline$\frac{1}{P_{1} \times \mathbf{P}_{5}}$ & 12.22 & 12.22 & $36.50^{* * *}$ & $36.50^{* * *}$ & $23.91 *$ & $23.91 *$ & -1.37 & -1.37 & 7.58 & $24.77^{* *}$ & 3.86 & 10.02 \\
\hline $\mathbf{P}_{1} \times \mathbf{P}_{6}$ & 13.17 & $32.29 * *$ & $40.59 * *$ & $22.80^{*}$ & $38.80^{* * *}$ & $27.72^{* *}$ & $24.40^{* * *}$ & $22.13^{* * *}$ & $30.17^{* *}$ & $50.97^{* * *}$ & $27.16^{* * *}$ & $34.70^{* *}$ \\
\hline $\mathbf{P}_{1} \times \mathbf{P}_{7}$ & 1.53 & 12.41 & $71.66^{* * *}$ & $49.93 * *$ & $46.58^{* * *}$ & $30.48^{* * *}$ & 1.24 & 12.27 & $17.25^{* * *}$ & $41.34 * *$ & 8.54 & $24.94 * *$ \\
\hline $\mathbf{P}_{1} \times \mathbf{P}_{8}$ & 14.24 & $37.62 * *$ & $-20.69^{* *}$ & -4.06 & -2.62 & 17.55 & 24.21 *** & $40.34 * *$ & $14.87^{*}$ & $33.22^{* * *}$ & $29.55^{* * *}$ & $37.24 * *$ \\
\hline $\mathbf{P}_{1} \times \mathbf{P}_{9}$ & 0.50 & $30.21 * *$ & $22.44 *$ & $23.64 *$ & 9.71 & $27.05 * *$ & 0.95 & $18.28 *$ & $16.62 *$ & $35.25 * *$ & $18.64 *$ & $25.67 * *$ \\
\hline $\mathbf{P}_{1} \times \mathbf{P}_{10}$ & 3.24 & $22.09 *$ & 10.65 & -3.36 & $18.62 *$ & 9.83 & $22.54 * *$ & $29.50^{* * *}$ & $33.09^{* *}$ & $54.35^{* * *}$ & $32.02^{* * *}$ & $40.33^{* *}$ \\
\hline $\mathbf{P}_{2} \times \mathbf{P}_{3}$ & 5.28 & -9.29 & $56.03^{* *}$ & $41.19^{* *}$ & $30.34^{* *}$ & 15.02 & $50.80^{* * *}$ & $35.05^{* * *}$ & $34.40^{* * *}$ & $38.72^{* * *}$ & $49.42^{* * *}$ & $36.65^{* * *}$ \\
\hline $\mathbf{P}_{2} \times \mathbf{P}_{4}$ & $90.07^{* * *}$ & $53.02 * *$ & $35.56^{* * *}$ & 15.17 & $63.08^{* * *}$ & $34.79^{* * *}$ & $61.89^{* * *}$ & $36.42^{* *}$ & $51.68^{\text {*** }}$ & $56.55^{* * *}$ & $58.76^{* * *}$ & $45.19^{* *}$ \\
\hline $\mathbf{P}_{2} \times \mathbf{P}_{5}$ & $70.37 * *$ & $70.37 * *$ & 15.59 & 15.59 & $43.99^{* * *}$ & $43.99 * *$ & $24.48^{* * *}$ & $24.48 * *$ & 43.65 *** & $48.27 * *$ & $34.84^{* * *}$ & $34.84 * *$ \\
\hline $\mathbf{P}_{2} \times \mathbf{P}_{6}$ & -4.34 & 11.83 & $40.49^{* * *}$ & $19.37 *$ & $25.48^{*}$ & 15.46 & 10.46 & 4.11 & $16.34 *$ & $21.56^{* *}$ & $\frac{3.07}{13.17}$ & $\frac{11.04}{11.71}$ \\
\hline $\mathbf{P}_{2} \times \mathbf{P}_{7}$ & 9.27 & 20.99 & $69.05^{* * *}$ & $43.64 * *$ & $48.16^{* * *}$ & $31.90^{* * *}$ & -9.77 & 0.07 & 3.23 & $24.43^{* * *}$ & -3.84 & 10.68 \\
\hline $\mathbf{P}_{2} \times \mathbf{P}_{8}$ & 11.81 & $34.70 * *$ & -12.31 & 6.08 & 0.17 & $20.92^{*}$ & 11.61 & $26.11^{* * *}$ & $39.07^{* * *}$ & $43.53^{* * *}$ & $34.49^{* * *}$ & $33.70^{* * *}$ \\
\hline $\mathbf{P}_{2} \times \mathbf{P}_{9}$ & $19.11^{*}$ & $54.32 * *$ & 4.36 & 5.38 & 12.91 & $30.75^{* * *}$ & $14.87 *$ & $34.60^{* * *}$ & $37.51^{\text {*** }}$ & $41.93^{* * *}$ & $36.23^{* * *}$ & $37.79^{* * *}$ \\
\hline $\mathbf{P}_{2} \times \mathbf{P}_{10}$ & $35.66^{* * *}$ & $60.43^{* *} *$ & $59.34^{* * *}$ & $35.38^{* * *}$ & $60.24 * *$ & $48.37 * *$ & $30.20^{* * *}$ & $37.60^{* *}$ & $38.28 * *$ & $48.10^{* *}$ & $33.75^{* * *}$ & $42.17^{* * *}$ \\
\hline $\mathbf{P}_{3} \times \mathbf{P}_{4}$ & $54.37^{* * *}$ & 33.01 ** & 70.02 ** & $53.85^{* * *}$ & $62.10^{* * *}$ & $43.04 * *$ & $33.75^{* * *}$ & $19.78^{*}$ & $63.49^{* * *}$ & $50.63^{* * *}$ & $48.24^{* * *}$ & $33.22^{* *}$ \\
\hline $\mathbf{P}_{3} \times \mathbf{P}_{5}$ & $29.11^{* *}$ & $29.11^{* *}$ & 8.53 & 8.53 & $19.20^{*}$ & 19.20 & -1.96 & -1.96 & $22.23^{* * *}$ & $22.23^{* * *}$ & 8.58 & 8.58 \\
\hline$\frac{\mathbf{P}_{3} \times \mathbf{Y}_{5}}{\mathbf{P}_{3} \times \mathbf{P}_{6}}$ & $44.11 * * *$ & $69.01^{* *}$ & $\frac{0.03}{35.08^{* * *}}$ & $22.24^{*}$ & $59.19^{* * *}$ & $\frac{19.20}{46.48 * *}$ & $\frac{1.00}{44.53 * *}$ & $\frac{-1.00}{36.23 * *}$ & $32.36^{* *}$ & $38.29^{* *}$ & 0... & 37.130** \\
\hline $\mathbf{P}_{3} \times \mathbf{P}_{7}$ & $52.00^{* * *}$ & $68.29^{* *}$ & $70.79^{* * *}$ & $54.55^{* *}$ & $81.61^{* *}$ & $61.67 * *$ & 9.36 & $21.28 * *$ & $24.89^{* *}$ & $50.55^{* *}$ & $16.45^{*}$ & $34.03^{* *}$ \\
\hline $\mathbf{P}_{3} \times \mathbf{P}_{8}$ & 8.79 & $31.06^{* *}$ & 14.74 & $38.81 * *$ & 11.66 & $34.79 * *$ & 9.01 & $23.17^{* * *}$ & $63.48^{\text {*** }}$ & $47.59^{* * *}$ & $34.61^{* * *}$ & $33.81^{* * *}$ \\
\hline$\frac{13 \times 18}{\mathbf{P}_{3} \times \mathbf{P}_{9}}$ & $29.34^{* * *}$ & $67.58^{* * *}$ & $24.65^{*}$ & $25.87^{* * *}$ & $27.37^{* * *}$ & $47.49^{* * *}$ & $37.21^{* * *}$ & $60.77^{* * *}$ & $99.44 * *$ & $80.05^{* * *}$ & $67.26 * *$ & $69.17^{* * *}$ \\
\hline$\frac{13 \times 19}{\mathbf{P}_{3} \times \mathbf{P}_{10}}$ & $\frac{27.54}{27.53^{* *}}$ & $50.81^{* *}$ & $\frac{24.0 \mathrm{~J}}{97.99^{* *}}$ & $\frac{3.01}{79.16^{* *}}$ & $77.63^{* * *}$ & $\frac{17.49}{64.47 * *}$ & $\frac{1.21}{20.20^{* *}}$ & $27.02 * *$ & $26.28 * *$ & $35.25^{* * *}$ & $22.87 * *$ & $30.61^{* *}$ \\
\hline $\mathbf{P}_{4} \times \mathbf{P}_{5}$ & 8.45 & 8.45 & 14.55 & 14.55 & 11.38 & 11.38 & $51.31 * *$ & $51.31 * *$ & $72.78^{* *}$ & $72.78^{* *}$ & $60.66^{* *}$ & $60.66 * *$ \\
\hline $\mathbf{P} \times \mathbf{P}$ & 5.11 & $22.87 *$ & $32.52 * *$ & 6.85 & $25.15^{*}$ & 15.16 & $22.30^{* *}$ & 15.27 & $18.93 *$ & $24.26^{* *}$ & $20.75^{*}$ & $19.19 *$ \\
\hline $\mathbf{P}_{4} \times \mathbf{P}_{7}$ & $31.92^{* * *}$ & $46.07 * *$ & $107.29^{* * *}$ & $67.13^{* * *}$ & 75.48 & $56.21 * *$ & $23.66^{* *}$ & $37.14 * *$ & $\frac{10.95}{26.44 * *}$ & $\frac{24.20}{52.41 * *}$ & $\frac{20.15}{24.93 *}$ & $\frac{19.19}{43.79 * *}$ \\
\hline $\mathbf{P}_{4} \times \mathbf{P}_{8}$ & -6.69 & 12.41 & $-29.71 * *$ & -14.97 & $-17.80^{*}$ & -0.77 & -6.53 & 5.61 & $25.05^{* *}$ & 15.22 & 10.45 & 9.80 \\
\hline $\mathbf{P}_{4} \times \mathbf{P}_{9}$ & -10.63 & 15.79 & $21.47^{*}$ & $22.66^{*}$ & 2.85 & 19.10 & 2.84 & $20.50^{*}$ & $56.79^{* * *}$ & $\frac{10.22 *}{44.46 *}$ & $29.46^{* * *}$ & $30.94^{* * *}$ \\
\hline $\mathbf{P}_{4} \times \mathbf{P}_{10}$ & 13.19 & $33.85^{* * *}$ & $35.13^{* * *}$ & 8.95 & $31.61^{* * *}$ & $21.86^{*}$ & $23.35 * *$ & $30.35^{* * *}$ & $56.43^{* * *}$ & $67.54^{* * *}$ & $37.87^{\text {*** }}$ & $46.56^{* * *}$ \\
\hline$\frac{4}{\mathbf{P}_{5} \times \mathbf{P}_{6}}$ & $47.19^{* * *}$ & $72.06^{* * *}$ & $26.92 * *$ & $26.92 * *$ & $50.32^{* * *}$ & $50.32 * *$ & $27.28^{* * * *}$ & $27.28^{* * *}$ & $56.15^{* * *}$ & $63.14^{* * *}$ & $42.91^{\text {*** }}$ & $42.91^{* * *}$ \\
\hline $\mathbf{P}_{5} \times \mathbf{P}_{7}$ & $25.65^{* *}$ & $39.12^{* * *}$ & $53.78^{* * *}$ & $53.78^{* *}$ & $46.18^{* *}$ & $46.18^{* * *}$ & 5.12 & $16.58^{*}$ & $13.32^{*}$ & $36.60^{* *}$ & 8.86 & $25.30^{* *}$ \\
\hline $\mathbf{P}_{5} \times \mathbf{P}_{8}$ & $22.33^{*}$ & $47.37^{* * *}$ & $-25.26^{* *}$ & -9.58 & -0.64 & 19.94 & $38.07^{* * * *}$ & $56.01^{* * *}$ & $75.49^{* * *}$ & $75.49^{* * *}$ & $64.49^{* * *}$ & $64.49^{* * *}$ \\
\hline $\mathbf{P}_{5} \times \mathbf{P}_{9}$ & $-33.75 * *$ & -14.17 & $80.06^{* * *}$ & $81.82^{* * *}$ & 14.05 & $32.06^{* * *}$ & $\frac{1.07}{-1.67}$ & 15.21 & $43.20^{* * *}$ & $43.20 * *$ & $25.97 * *$ & $27.40^{* * *}$ \\
\hline $\begin{array}{l}\mathbf{r}_{5} \times \mathbf{r}_{9} \\
\mathbf{P}_{5} \times \mathbf{P}_{10}\end{array}$ & 9.34 & $29.30^{* *}$ & $68.95^{* * *}$ & $68.95^{* *}$ & $48.40^{* * *}$ & $48.40^{* * *}$ & $\begin{array}{l}-1.07 \\
19.70^{*}\end{array}$ & $26.50^{* *}$ & $32.44 * *$ & $41.84 * *$ & 25.29 & $33.19^{* *}$ \\
\hline$\frac{\mathbf{5}_{5} \times \mathbf{1}_{10}}{\mathbf{P}_{6} \times \mathbf{P}_{7}}$ & 18.18 & $38.14^{* * *}$ & $22.79 *$ & $-19.37^{*}$ & $20.02 *$ & $\frac{7.40}{10.44}$ & $15.95^{*}$ & $28.59^{* *}$ & $34.85^{* *}$ & $62.55^{* * *}$ & $24.58^{* * *}$ & $43.39^{* *}$ \\
\hline $\mathbf{P}_{6} \times \mathbf{P}_{8}$ & 17.21 & $41.20^{* * *}$ & -2.66 & 17.76 & 7.62 & $29.91 * *$ & 11.73 & $26.24 * *$ & $32.85^{* * *}$ & $38.80^{* * *}$ & $32.49^{* * *}$ & $31.71^{* * *}$ \\
\hline $\mathbf{P}_{6} \times \mathbf{P}_{9}$ & $39.67 * *$ & $80.96 * *$ & $40.37^{*}$ & $41.75 * *$ & $39.97 * *$ & $62.07 * *$ & $33.37 * *$ & $56.27 * *$ & $69.74 * *$ & $77.35^{* * *}$ & $63.58^{* * *}$ & $65.45^{* * *}$ \\
\hline $\mathbf{P}_{6} \times \mathbf{P}_{10}$ & $18.85^{*}$ & $40.55^{* * *}$ & $24.76^{*}$ & -18.60 & $21.03^{*}$ & 12.06 & $26.87 * *$ & $34.07 * *$ & $57.85^{* * *}$ & $69.06^{* *}$ & $40.47^{* * *}$ & $49.32^{* * *}$ \\
\hline $\mathbf{P}_{7} \times \mathbf{P}_{8}$ & $32.74 * *$ & $59.91 * *$ & 3.82 & $25.59 * *$ & $18.78^{*}$ & $43.38^{* * *}$ & $18.89^{* * *}$ & $34.33 * *$ & $21.60^{* *}$ & $46.58 * *$ & $21.34 * *$ & $39.67 * *$ \\
\hline $\mathbf{P}_{7} \times \mathbf{P}_{9}$ & $20.36^{*}$ & $55.95 * *$ & $22.85^{*}$ & 24.06 & $21.41^{*}$ & $40.59 * *$ & $23.45^{* * *}$ & $44.65 * *$ & $43.13 * *$ & $72.53^{* *}$ & $36.22^{* * *}$ & $56.80^{* * *}$ \\
\hline $\mathbf{P}_{7} \times \mathbf{P}_{10}$ & 6.04 & $25.41 *$ & $99.36^{* * *}$ & $30.91 * *$ & $38.30^{* * *}$ & $28.06^{* *}$ & 10.89 & $22.98^{* * *}$ & $14.73^{*}$ & $38.29 * *$ & 12.64 & $29.65^{* * *}$ \\
\hline$\frac{17 \times 10}{\mathbf{P}_{8} \times \mathbf{P}_{9}}$ & $\frac{0.04}{7.37}$ & $39.12^{* * *}$ & $\frac{0.69}{-0.09}$ & $\frac{10 . \% 1}{20.14 *}$ & 7.67 & $29.98 * *$ & 8.97 & $27.68 * *$ & $53.10^{* * *}$ & $25.27^{* *}$ & $25.20^{* *}$ & $26.63^{* * *}$ \\
\hline$\frac{18 \times 19}{\mathbf{P}_{8} \times \mathbf{P}_{10}}$ & $28.80^{\text {*** }}$ & $55.17^{* * *}$ & 5.43 & $27.55^{* *}$ & $17.52^{*}$ & $41.87 * *$ & $31.48^{* *}$ & $48.56^{* *}$ & $31.49^{* *}$ & $40.83^{* *}$ & $36.59^{* *}$ & $45.19^{* * *}$ \\
\hline $\mathbf{P}_{9} \times \mathbf{P}_{10}$ & -15.15 & 9.94 & 8.73 & 9.79 & -5.12 & 9.87 & -4.57 & 11.81 & $14.36^{*}$ & $22.49 * *$ & 9.56 & $16.46^{*}$ \\
\hline $\begin{array}{c}\text { No. of crosses with } \\
\text { significant }(+) \text { heterosis }\end{array}$ & 22 & 34 & 30 & 28 & 31 & 32 & 28 & 36 & 43 & 44 & 35 & 40 \\
\hline $\begin{array}{l}\text { No. of crosses with } \\
\text { significant (-) 'heterosis }\end{array}$ & 1 & 0 & 3 & 2 & 1 & 0 & 0 & 0 & 0 & 0 & 0 & 0 \\
\hline Range of heterosis & -33.75 to 90.07 & -14.17 to 80.96 & \begin{tabular}{|c|}
-29.71 to 99.36 \\
\end{tabular} & -18.60 to 79.16 & -17.80 to 81.61 & $\begin{array}{c}-0.77 \text { to } 64.47 \\
\end{array}$ & -9.77 to 61.89 & -1.96 to 60.77 & 3.23 to 99.44 & 15.22 to 80.05 & -3.84 to 67.26 & 9.80 to 69.17 \\
\hline
\end{tabular}

*, ** Significant at 5 per cent and 1 per cent probability levels, respectively. 
Table.1 Cont.....

\begin{tabular}{|c|c|c|c|c|c|c|c|c|c|c|c|c|}
\hline \multirow{3}{*}{ Crosses } & \multicolumn{6}{|c|}{ Total soluble solids (TSS) } & \multicolumn{6}{|c|}{ Ascorbic acid (mg/100 g fresh fruit) } \\
\hline & \multicolumn{2}{|c|}{$\mathbf{E}_{1}$} & \multicolumn{2}{|c|}{$\mathbf{E}_{2}$} & \multicolumn{2}{|c|}{ Pooled } & \multicolumn{2}{|c|}{$\mathbf{E}_{1}$} & \multicolumn{2}{|c|}{$\mathbf{E}_{2}$} & \multicolumn{2}{|c|}{ Pooled } \\
\hline & BP & SV & BP & SV & $\mathbf{B P}$ & SV & BP & SV & $\mathbf{B P}$ & SV & BP & SV \\
\hline$\overline{\mathbf{P}_{1} \times \mathbf{P}_{2}}$ & $26.81 * *$ & $62.86^{* *}$ & $16.76^{* * *}$ & $46.74^{* *}$ & 21.59 ** & $54.39 * *$ & $4.70^{*}$ & $8.31 * *$ & 3.94 & $8.78^{* * *}$ & 4.32 & $8.54 * *$ \\
\hline $\mathbf{P}_{1} \times \mathbf{P}_{3}$ & $15.28 * *$ & $62.26 * *$ & $15.22 * *$ & $57.34 * *$ & $15.25 * *$ & $59.67 * *$ & $-13.73^{* * *}$ & $-10.76^{* *}$ & -2.81 & 1.72 & $-8.32 * *$ & -4.61 \\
\hline $\mathbf{P}_{1} \times \mathbf{P}_{4}$ & $17.77^{* *}$ & $68.42 * *$ & $-12.45^{*}$ & $21.33^{* * *}$ & 2.13 & $43.68 * *$ & -4.18 & $6.16^{*}$ & $-18.75^{* * *}$ & $-9.73 * *$ & $-11.37 * *$ & -1.67 \\
\hline $\mathbf{P}_{1} \times \mathbf{P}_{5}$ & $-8.67^{*}$ & 17.29 ** & $31.68^{* * *}$ & $65.49^{* *}$ & $12.31^{*}$ & $42.61^{* * *}$ & 2.83 & $6.38^{* * *}$ & 1.17 & $5.88^{*}$ & 2.01 & $6.13^{*}$ \\
\hline $\mathbf{P}_{1} \times \mathbf{P}_{6}$ & -5.48 & $40.15 * *$ & -2.56 & $39.67 * *$ & -3.97 & $39.90 * *$ & -4.40 & -0.82 & $-6.16^{* *}$ & -0.61 & $-5.28^{*}$ & -0.71 \\
\hline $\mathbf{P}_{1} \times \mathbf{P}_{7}$ & $13.73^{* * *}$ & $66.92^{* *}$ & $22.30^{* *}$ & $73.64 * *$ & $18.16^{* * *}$ & $70.45^{* * *}$ & 2.80 & $6.34 * *$ & $-9.95^{* *}$ & $-5.76^{*}$ & -3.52 & 0.38 \\
\hline $\mathbf{P}_{1} \times \mathbf{P}_{8}$ & 7.10 & $58.80^{* * *}$ & -2.10 & $39.67^{* *}$ & 2.36 & $48.75 * *$ & -2.33 & $5.75^{*}$ & -0.15 & 4.50 & -1.24 & $5.13^{*}$ \\
\hline $\mathbf{P}_{1} \times \mathbf{P}_{9}$ & 3.52 & $50.38^{* * *}$ & 2.22 & $43.75^{* * *}$ & 2.85 & $46.90^{* * *}$ & $-9.11^{* * *}$ & 2.89 & $-9.07 * *$ & 3.28 & $-9.09 * *$ & 3.08 \\
\hline $\mathbf{P}_{1} \times \mathbf{P}_{10}$ & -3.15 & $80.15^{* * *}$ & $-18.88 * *$ & $38.32^{* * *}$ & $-11.08 * *$ & $58.17 * *$ & -3.05 & 0.30 & $6.20 * *$ & $11.14^{* * *}$ & 1.54 & $5.64 *$ \\
\hline $\mathbf{P}_{2} \times \mathbf{P}_{3}$ & -0.11 & $40.60^{* * *}$ & 0.80 & $37.64 * *$ & 0.36 & $39.04 * *$ & -3.58 & $-10.98^{* *}$ & -0.75 & $-8.58^{* * *}$ & -2.18 & $-9.80^{* * *}$ \\
\hline $\mathbf{P}_{2} \times \mathbf{P}_{4}$ & 0.95 & $44.36^{* *}$ & -3.63 & $33.56^{* *}$ & -1.42 & $38.69 * *$ & -4.29 & $6.05^{*}$ & $-5.60^{*}$ & $4.88 *$ & $-4.93^{*}$ & $5.47^{*}$ \\
\hline $\mathbf{P}_{2} \times \mathbf{P}_{5}$ & 3.42 & $31.73^{* *}$ & 8.95 & $35.60^{* *}$ & 6.30 & $33.76^{* *}$ & 3.89 & 3.89 & $8.09 * *$ & $8.09 * *$ & $5.96^{*}$ & $5.96^{*}$ \\
\hline $\mathbf{P}_{2} \times \mathbf{P}_{6}$ & -3.45 & $43.16^{* *}$ & -3.51 & $38.32 * *$ & -3.48 & $40.61 * *$ & 2.32 & $6.16^{*}$ & $7.28 * *$ & $13.62 * *$ & $4.79 *$ & $9.84 * *$ \\
\hline $\mathbf{P}_{2} \times \mathbf{P}_{7}$ & -3.69 & $41.35^{* * *}$ & $-11.20^{*}$ & $26.09^{* *}$ & -7.57 & $33.33^{* * *}$ & 2.49 & -0.74 & 3.59 & 0.23 & 3.03 & -0.26 \\
\hline $\mathbf{P}_{2} \times \mathbf{P}_{8}$ & -0.61 & $47.37 * *$ & 0.76 & $43.75 * *$ & 0.10 & $45.47 * *$ & -1.54 & $6.60^{* *}$ & $8.54 * *$ & $13.51^{* * *}$ & 3.34 & $10.01^{* * *}$ \\
\hline $\mathbf{P}_{2} \times \mathbf{P}_{9}$ & $9.32 *$ & $58.80 * *$ & $-27.73^{* *}$ & 1.63 & $-9.85^{*}$ & $28.77 * *$ & 0.59 & $13.87 * *$ & -0.97 & $12.48^{* * *}$ & -0.18 & $13.18^{* * *}$ \\
\hline $\mathbf{P}_{2} \times \mathbf{P}_{1}$ & $-8.25^{* *}$ & $70.68^{* *}$ & $-14.90^{* * *}$ & $45.11 * *$ & $-11.60^{* * *}$ & $57.24 * *$ & 0.87 & -1.00 & 1.13 & -0.80 & 1.00 & -0.90 \\
\hline $\mathbf{P}_{3} \times \mathbf{P}_{4}$ & $13.56^{* * *}$ & $62.41^{* *}$ & -0.20 & $38.32^{* *}$ & 6.44 & $49.75^{* *}$ & $-20.62 * *$ & $-12.05^{* *}$ & 0.17 & $11.29^{* *}$ & $-10.36^{* * *}$ & -0.55 \\
\hline $\mathbf{P}_{3} \times \mathbf{P}_{5}$ & 2.56 & $44.36^{* *}$ & $-14.63 * *$ & $16.58 *$ & -6.34 & $29.76^{* *}$ & $-5.38^{*}$ & $-5.38^{*}$ & $-5.30 *$ & $-5.30^{*}$ & $-5.34 *$ & $-5.34 *$ \\
\hline $\mathbf{P}_{3} \times \mathbf{P}_{6}$ & 5.48 & 56.39 ** & 1.23 & $45.11^{* * *}$ & 3.28 & $50.46 * *$ & $-15.80 * *$ & $-12.65^{* *}$ & 0.76 & $6.71 * *$ & $-7.55 * *$ & -3.10 \\
\hline $\mathbf{P}_{3} \times \mathbf{P}_{7}$ & 2.46 & $50.38^{* * *}$ & 3.16 & $46.47 * *$ & 2.82 & $48.32 * *$ & -0.88 & -4.01 & $-5.28^{*}$ & $-8.36 * *$ & -3.05 & $-6.15^{*}$ \\
\hline $\mathbf{P}_{3} \times \mathbf{P}_{8}$ & -3.65 & $42.86^{* *}$ & -2.10 & $39.67^{* *}$ & -2.85 & $41.18^{* *}$ & $-15.18^{* *}$ & $-8.16^{* * *}$ & -2.44 & 2.02 & $-9.01 * *$ & -3.14 \\
\hline $\mathbf{P}_{3} \times \mathbf{P}_{9}$ & $7.66^{*}$ & $56.39 * *$ & $14.78^{* * *}$ & $61.41^{* * *}$ & $11.34^{*}$ & $59.03^{* * *}$ & $-14.52^{* *}$ & -3.23 & $-20.36^{* * *}$ & $-9.54 * *$ & $-17.40^{* *}$ & $-6.34 * *$ \\
\hline $\mathbf{P}_{3} \times \mathbf{P}_{10}$ & $-13.50^{* * *}$ & $60.90^{* * *}$ & $-27.25 * *$ & $24.05^{* *}$ & $-20.43 * *$ & $41.54 * *$ & $15.27 * *$ & $13.13^{* * *}$ & -1.05 & -2.94 & $7.23 * *$ & $5.21 *$ \\
\hline $\mathbf{P}_{4} \times \mathbf{P}_{5}$ & $-16.51 * *$ & $19.40 * *$ & $-15.88 * *$ & $16.58^{*}$ & $-16.18^{* * *}$ & $17.92 * *$ & $-13.02 * *$ & -3.64 & -3.74 & $6.94 * *$ & $-8.44 * *$ & 1.58 \\
\hline $\mathbf{P}_{4} \times \mathbf{P}_{6}$ & $10.04 * *$ & $63.16^{* *}$ & 7.58 & $54.21^{* *}$ & $8.77^{*}$ & $58.46 * *$ & $-11.22 * *$ & -1.63 & $-8.69^{* *}$ & 1.45 & $-9.97 * *$ & -0.11 \\
\hline $\mathbf{P}_{4} \times \mathbf{P}_{7}$ & 1.43 & $48.87^{* * *}$ & $-12.25^{*}$ & 24.59 ** & -5.64 & $36.12^{* * *}$ & 2.41 & $13.46^{* * *}$ & -2.16 & $8.70^{* * *}$ & 0.15 & $11.12^{* * *}$ \\
\hline $\mathbf{P}_{4} \times \mathbf{P}_{9}$ & $-13.66^{* *}$ & $25.41^{* *}$ & $-11.88^{*}$ & $23.91^{* *}$ & $-12.74 * *$ & $24.63 * *$ & 0.46 & $13.72^{* * *}$ & -1.68 & $11.67^{* *}$ & -0.60 & $12.71^{* * *}$ \\
\hline $\mathbf{P}_{4} \times \mathbf{P}_{10}$ & $-6.22^{*}$ & $74.44^{* * *}$ & -1.35 & $68.21^{* *}$ & -3.77 & $71.16^{* * *}$ & $\frac{0.76}{-0.27}$ & $10.50^{* * *}$ & -0.31 & $10.76^{* * *}$ & -0.29 & $10.63^{* *}$ \\
\hline $\mathbf{P}_{5} \times \mathbf{P}_{6}$ & $-11.26^{* * *}$ & $31.58^{* *}$ & $17.35^{\text {*** }}$ & $68.21^{* *}$ & 3.53 & $50.82^{* * *}$ & -4.58 & -1.00 & 0.86 & $6.83^{* * *}$ & -1.87 & 2.86 \\
\hline $\mathbf{P}_{5} \times \mathbf{P}_{7}$ & $-14.96^{* *}$ & $24.81^{* *}$ & -4.50 & $35.60^{\text {*** }}$ & $-9.55^{*}$ & $30.48 * *$ & $7.90^{* * *}$ & $7.90^{* * *}$ & $9.88^{* * *}$ & $9.88^{* * *}$ & $8.88^{* * *}$ & $8.88 * *$ \\
\hline $\mathbf{P}_{5} \times \mathbf{P}_{8}$ & $-17.85^{* * *}$ & $21.80^{* * *}$ & 5.71 & $50.82^{* * *}$ & -5.70 & $37.04 * *$ & -4.01 & 3.93 & -3.94 & 0.46 & -3.98 & 2.22 \\
\hline $\mathbf{P}_{5} \times \mathbf{P}_{9}$ & $-21.33^{* * *}$ & $14.29^{*}$ & $-17.10^{* * *}$ & $16.58^{*}$ & $-19.14^{* * *}$ & $15.49^{*}$ & $-12.12^{* * *}$ & -0.52 & $-12.23^{* * *}$ & $\frac{0.40}{-0.31}$ & $-12.17^{* *}$ & -0.41 \\
\hline $\mathbf{P}_{5} \times \mathbf{P}_{10}$ & $-14.31^{* * *}$ & $59.40^{* *}$ & $-37.21^{* *}$ & 7.07 & $-25.84 * *$ & $31.91 * *$ & $15.21^{* *}$ & $15.21 * *$ & -0.88 & -0.88 & $7.28^{* *}$ & $7.28^{* * *}$ \\
\hline $\mathbf{P}_{6} \times \mathbf{P}_{7}$ & $-10.75^{* * *}$ & $32.33^{* *}$ & -8.72 & $30.84 * *$ & $-9.70 *$ & $31.55^{* *}$ & $6.79 * *$ & $10.79 * *$ & 3.49 & $9.61 * *$ & $5.15^{*}$ & $10.21 * *$ \\
\hline $\mathbf{P}_{6} \times \mathbf{P}_{8}$ & 5.58 & $56.54 * *$ & 6.07 & $52.04 * *$ & 5.83 & $54.18^{* * *}$ & $-6.20^{* * *}$ & 1.56 & -3.85 & 1.83 & $-4.47^{*}$ & 1.69 \\
\hline $\mathbf{P}_{6} \times \mathbf{P}_{9}$ & $-17.34^{* * *}$ & $22.56^{* * *}$ & $13.55^{\text {*** }}$ & $62.77^{* * *}$ & -1.37 & $43.68^{* * *}$ & $-20.22^{* *}$ & $-9.68^{* * * *}$ & $-6.18^{* * *}$ & $6.56 * *$ & $-13.29 * *$ & -1.67 \\
\hline $\mathbf{P}_{6} \times \mathbf{P}_{10}$ & $-9.86^{* *}$ & $67.67^{* * *}$ & $\begin{array}{l}-7.09 \\
\end{array}$ & $58.42^{* * *}$ & $-8.47 *$ & $62.81^{* * *}$ & -3.58 & 0.04 & $-5.33^{*}$ & 0.27 & -4.45 & 0.15 \\
\hline $\mathbf{P}_{7} \times \mathbf{P}_{8}$ & $11.66^{* *}$ & $65.56^{* * *}$ & $12.29 *$ & $60.19^{* *}$ & $11.98^{* * *}$ & $62.74 * *$ & -1.95 & $6.16^{*}$ & 1.90 & $6.56 * *$ & -0.09 & $6.36 * *$ \\
\hline $\mathbf{P}_{7} \times \mathbf{P}_{9}$ & $8.30^{*}$ & $58.95 * *$ & $11.29 *$ & $58.02^{* * *}$ & $9.85^{*}$ & $58.46 * *$ & $-10.62 * *$ & 1.19 & $-9.88^{* * *}$ & 2.37 & $-10.25 * *$ & 1.77 \\
\hline $\mathbf{P}_{7} \times \mathbf{P}_{10}$ & $\begin{array}{c}0.50 \\
-16.57 * *\end{array}$ & $55.19^{* *}$ & $-18.09 * *$ & $39.67 * *$ & $\frac{1.05}{-17.34 * *}$ & $\frac{J .40}{47.04 * *}$ & $6.65 * *$ & 4.67 & $12.37 * *$ & $10.23^{* *}$ & $9.47 * *$ & $7.41^{1 * *}$ \\
\hline $\mathbf{P}_{8} \times \mathbf{P}_{9}$ & 1.83 & $50.98^{* * *}$ & 3.05 & $47.01^{* *}$ & 2.46 & $48.89 * *$ & -0.43 & $12.72^{* * *}$ & -3.90 & $9.16^{* * *}$ & -2.14 & $10.96^{* *}$ \\
\hline $\mathbf{P}_{8} \times \mathbf{P}_{10}$ & $-10.99^{* * *}$ & $65.56^{* * *}$ & -6.06 & $60.19^{* *}$ & $-8.51^{*}$ & $62.74 * *$ & 2.16 & $10.61 * *$ & -0.29 & 4.27 & 0.97 & $7.49 * *$ \\
\hline $\mathbf{P}_{9} \times \mathbf{P}_{10}$ & $-12.69^{* * *}$ & $62.41^{* * *}$ & $-38.01 * *$ & 5.71 & $-25.44 * *$ & $32.62 * *$ & $-8.16^{* * *}$ & 3.97 & $-9.47^{* * *}$ & 2.82 & $-8.81^{* * *}$ & 3.40 \\
\hline $\begin{array}{c}\text { No. of crosses with } \\
\text { significant }(+) \text { heterosis }\end{array}$ & 10 & 45 & 10 & 42 & 9 & 45 & 6 & 20 & 6 & 22 & 7 & 21 \\
\hline $\begin{array}{c}\text { No. of crosses with } \\
\text { significant (-) 'heterosis }\end{array}$ & 16 & 0 & 14 & 0 & 14 & 0 & 15 & 7 & 15 & 6 & 18 & 4 \\
\hline Range of heterosis & -17.85 to 26.81 & $\begin{array}{l}-14.29 \text { to } 74.44 \\
\end{array}$ & $\begin{array}{l}-38.01 \text { to } 31.68 \\
\end{array}$ & 1.63 to 73.64 & -25.84 to 21.59 & 15.49 to -71.16 & -20.62 to 15.27 & -12.65 to 15.21 & -20.36 to 12.37 & -9.73 to 11.67 & -13.29 to 9.47 & $\begin{array}{l}-9.80 \text { to } 13.18 \\
\end{array}$ \\
\hline
\end{tabular}

$$
\text { *, ** Significant at } 5 \text { per cent and } 1 \text { per cent probability levels, respectively. }
$$


Table.1 Cont.....

\begin{tabular}{|c|c|c|c|c|c|c|c|c|c|c|c|c|}
\hline \multirow{3}{*}{ Crosses } & \multicolumn{6}{|c|}{ Reducing sugar (\%) } & \multicolumn{6}{|c|}{ Non-reducing sugar $(\%)$} \\
\hline & \multicolumn{2}{|c|}{ ENV1 } & \multicolumn{2}{|c|}{ ENV2 } & \multicolumn{2}{|c|}{ Pooled } & \multicolumn{2}{|c|}{ ENV1 } & \multicolumn{2}{|c|}{ ENV2 } & \multicolumn{2}{|c|}{ Pooled } \\
\hline & BP & SV & BP & SV & BP & SV & BP & SV & BP & SV & BP & SV \\
\hline$\overline{P_{1} \times P_{2}}$ & $-22.42 * *$ & $5.41^{* *}$ & $-24.21 * *$ & $7.78^{* *}$ & $-23.26 * *$ & $6.50^{* * *}$ & $16.40 * *$ & $15.48 * *$ & $61.14 * *$ & $59.32 * *$ & $34.82 * *$ & $33.57 * *$ \\
\hline $\mathbf{P}_{1} \times \mathbf{P}_{3}$ & $24.11^{* * *}$ & $33.07^{* * *}$ & $29.35^{* * *}$ & $40.33^{* * *}$ & $26.53^{* * *}$ & $36.40^{* * *}$ & $-21.64 * *$ & $-16.67^{* *}$ & 1.04 & $10.17^{*}$ & $-12.15^{* * *}$ & -5.59 \\
\hline $\mathbf{P}_{1} \times \mathbf{P}_{4}$ & $3.93^{*}$ & $16.63^{* * *}$ & $31.75^{* * *}$ & $50.71^{* * *}$ & $16.84^{* * *}$ & $32.29 * *$ & $12.40 * *$ & $11.51^{* *}$ & $10.86^{*}$ & $9.60^{*}$ & $11.76^{* *}$ & $10.72^{* * *}$ \\
\hline $\mathbf{P}_{1} \times \mathbf{P}_{5}$ & $7.58 * *$ & $13.83^{* * *}$ & $25.17^{* * *}$ & $33.73^{* * *}$ & $15.70^{* * *}$ & $22.97 * *$ & $9.52 * *$ & $9.52^{* *}$ & $27.12^{* * *}$ & $27.12^{* * *}$ & $16.78^{* * *}$ & $16.78^{* * *}$ \\
\hline $\mathbf{P}_{1} \times \mathbf{P}_{6}$ & $33.14 * *$ & $40.88^{* * *}$ & $39.96 * *$ & $49.53 * *$ & $36.29 * *$ & $44.85 * *$ & $-21.40 * *$ & $-19.84 * *$ & 2.75 & 5.65 & $-11.39 * *$ & $-9.32 *$ \\
\hline $\mathbf{P}_{1} \times \mathbf{P}_{7}$ & $-8.63^{* *}$ & $16.63^{* *}$ & $-9.25 * *$ & 20.28 ** & $-8.92 * *$ & $18.31 * *$ & 5.60 & 4.76 & $48.57 * *$ & $46.89 * *$ & $23.29 * *$ & $22.14 * *$ \\
\hline $\mathbf{P}_{1} \times \mathbf{P}_{8}$ & $23.41^{* * *}$ & $32.06 * *$ & $28.54 * *$ & $39.15^{* * *}$ & $25.78^{* * *}$ & $35.32 * *$ & $-19.93 * *$ & $-12.30^{* * *}$ & 2.49 & $16.38^{* * *}$ & $-10.48^{* *}$ & -0.47 \\
\hline $\mathbf{P}_{1} \times \mathbf{P}_{9}$ & $20.08^{* * *}$ & $27.05^{* *}$ & $24.72^{* * *}$ & $33.25^{* * *}$ & $22.22^{* * *}$ & $29.90 * *$ & $-16.08 * *$ & -4.76 & 6.64 & $27.12^{* *}$ & $-6.44 *$ & $8.39 *$ \\
\hline $\mathbf{P}_{1} \times \mathbf{P}_{10}$ & $-6.45^{* * *}$ & $4.61^{*}$ & $-6.21^{*}$ & $6.84 *$ & $-6.34 * *$ & $5.63^{*}$ & $14.40^{* * *}$ & $13.49 * *$ & $62.86 * *$ & $61.02^{* * *}$ & $34.35^{* * *}$ & $33.10^{* * *}$ \\
\hline $\mathbf{P}_{2} \times \mathbf{P}_{3}$ & $-8.41^{* *}$ & $24.45^{* * *}$ & $-8.46^{* * *}$ & $30.19^{* * *}$ & $-8.43 * *$ & $27.09 * *$ & -3.73 & 2.38 & $25.91 * *$ & $37.29 * *$ & $8.68 *$ & $16.78^{* * *}$ \\
\hline $\mathbf{P}_{2} \times \mathbf{P}_{4}$ & $-12.83 * *$ & $18.44 * *$ & $-13.43 * *$ & $23.11^{* * *}$ & $-13.11 * *$ & $20.59 * *$ & $8.23^{*}$ & 4.37 & $47.62^{* * *}$ & $40.11^{* *}$ & $24.33^{* * *}$ & $19.11^{* * *}$ \\
\hline $\mathbf{P}_{2} \times \mathbf{P}_{5}$ & $-26.40^{* * *}$ & 0.00 & $-28.69^{* *}$ & 1.42 & $-27.48 * *$ & 0.65 & 5.95 & 5.95 & $42.37^{* *}$ & $42.37^{* * *}$ & $20.98^{* * *}$ & $20.98^{* * *}$ \\
\hline $\mathbf{P}_{2} \times \mathbf{P}_{6}$ & $-8.55^{* * *}$ & $24.25^{* *}$ & $-8.62^{* * *}$ & $29.95^{* *}$ & $-8.59 * *$ & $26.87^{* *}$ & -0.78 & 1.19 & $31.87 * *$ & $35.59 * *$ & $12.76^{* *}$ & $15.38^{* * *}$ \\
\hline $\mathbf{P}_{2} \times \mathbf{P}_{7}$ & $-7.52^{* * *}$ & $25.65 * *$ & $-7.46 * *$ & $31.60^{* * *}$ & $-7.49 * *$ & $28.39 * *$ & $24.24 * *$ & -2.38 & 87.80 ** & $30.51^{* *}$ & $48.60^{* *}$ & $11.19^{* * *}$ \\
\hline $\mathbf{P}_{2} \times \mathbf{P}_{8}$ & $-11.95 * *$ & $19.64 * *$ & $-12.44^{* *}$ & $24.53^{* * *}$ & $-12.18 * *$ & $21.89^{* * *}$ & -3.26 & 5.95 & $25.37 * *$ & $42.37^{* * *}$ & $8.81 * *$ & $20.98^{* * *}$ \\
\hline $\mathbf{P}_{2} \times \mathbf{P}_{9}$ & $-14.60 * *$ & $16.03^{* * *}$ & $-15.42 * *$ & $20.28^{* *}$ & $-14.99 * *$ & $17.98^{* * *}$ & -1.75 & $11.51^{* *}$ & $26.07 * *$ & $50.28^{* *}$ & $10.06^{* *}$ & $27.51^{* * *}$ \\
\hline $\mathbf{P}_{2} \times \mathbf{P}_{10}$ & $-7.52^{* * *}$ & $25.65 * *$ & $-7.46 * *$ & $31.60^{\text {*** }}$ & $-7.49 * *$ & $28.39 * *$ & 0.86 & $-7.14 *$ & 39.49 ** & $23.73^{* * *}$ & $16.45^{\text {*** }}$ & 5.59 \\
\hline $\mathbf{P}_{3} \times \mathbf{P}_{4}$ & $14.64 * *$ & $28.66^{* * *}$ & $18.14^{* * *}$ & $35.14 * *$ & $16.27^{* * *}$ & $31.64 * *$ & $-10.45 * *$ & -4.76 & $16.58^{* * *}$ & $27.12^{* *}$ & 0.87 & $8.39 *$ \\
\hline $\mathbf{P}_{3} \times \mathbf{P}_{5}$ & $36.82 * *$ & $46.69^{* * *}$ & $44.13^{* * *}$ & $56.37 * *$ & $40.20^{* * *}$ & $51.14 * *$ & $-35.45 * *$ & $-31.35^{* *}$ & $-18.13 * *$ & $-10.73^{*}$ & $-28.20^{* * *}$ & $-22.84 * *$ \\
\hline $\mathbf{P}_{3} \times \mathbf{P}_{6}$ & 2.06 & $9.42^{* * *}$ & 3.70 & $12.50^{* * *}$ & 2.81 & $10.83 * *$ & 5.60 & $12.30^{* * *}$ & $38.86^{* * *}$ & $51.41^{* * *}$ & $19.52^{* * *}$ & $28.44 * *$ \\
\hline $\mathbf{P}_{3} \times \mathbf{P}_{7}$ & $17.27 * *$ & $49.70^{* *}$ & $20.64 * *$ & $59.91 * *$ & $18.85^{* * *}$ & $54.39 * *$ & $-39.93 * *$ & $-36.11 * *$ & $-24.35 * *$ & $-17.51 * *$ & $-33.41 * *$ & $-28.44 * *$ \\
\hline $\mathbf{P}_{3} \times \mathbf{P}_{8}$ & $14.39^{* * *}$ & $22.65^{* * *}$ & $18.04 * *$ & $28.07 * *$ & $16.08^{* * *}$ & $25.14 * *$ & -5.43 & 3.57 & $22.39^{* *}$ & $38.98^{* * *}$ & 6.29 & $18.18^{* * *}$ \\
\hline $\mathbf{P}_{3} \times \mathbf{P}_{9}$ & $8.22 * *$ & $16.03 * *$ & $11.52^{* * *}$ & $20.99^{* *}$ & $9.75^{* *}$ & $18.31^{* *}$ & -3.85 & $9.13 * *$ & $18.01^{* * *}$ & $40.68^{* * *}$ & 5.43 & $22.14 * *$ \\
\hline $\mathbf{P}_{3} \times \mathbf{P}_{10}$ & -0.36 & $11.42^{* *}$ & 0.83 & $14.86 * *$ & 0.19 & $13.00^{* * *}$ & $7.84 * *$ & $14.68^{* * *}$ & $41.97 * *$ & $54.80^{* * *}$ & $22.13^{* * *}$ & $31.24 * *$ \\
\hline $\mathbf{P}_{4} \times \mathbf{P}_{5}$ & $13.57^{* * *}$ & $27.45^{* *}$ & 2.89 & $17.69 * *$ & $8.61 * *$ & $22.97^{* *}$ & -4.76 & -4.76 & $47.46^{* *}$ & $47.46^{* *}$ & $16.78^{* *}$ & $16.78^{* *}$ \\
\hline $\mathbf{P}_{4} \times \mathbf{P}_{6}$ & $20.89^{* * *}$ & $35.67 * *$ & $23.92 * *$ & $41.75^{* * *}$ & $22.30^{* * *}$ & $38.46 * *$ & $-10.89 * *$ & $-9.13^{* *}$ & 6.04 & $9.04 *$ & -3.87 & -1.63 \\
\hline $\mathbf{P}_{4} \times \mathbf{P}_{7}$ & $-18.52 * *$ & 4.01 & $-19.93^{* *}$ & $6.13^{*}$ & $-19.18 * *$ & $4.98 *$ & $27.16^{* * *}$ & $22.62 * *$ & $75.00 * *$ & $66.10^{* * *}$ & $46.72 * *$ & $40.56 * *$ \\
\hline $\mathbf{P}_{4} \times \mathbf{P}_{8}$ & $10.54 * *$ & $24.05^{* * *}$ & $13.40 * *$ & $29.72^{* * *}$ & $11.87^{* * *}$ & $26.65^{* * *}$ & $-7.97 * *$ & 0.79 & $18.91^{* * *}$ & $35.03^{* * *}$ & 3.35 & $14.92^{* * *}$ \\
\hline $\mathbf{P}_{4} \times \mathbf{P}_{9}$ & $10.71^{* *}$ & $24.25 * *$ & $13.61^{* * *}$ & $29.95^{* *}$ & $12.06^{* *}$ & $26.87 * *$ & -7.69 & 4.76 & $18.01^{* *}$ & $40.68^{* *}$ & 3.22 & $19.58^{* * *}$ \\
\hline $\mathbf{P}_{4} \times \mathbf{P}_{10}$ & $-6.07 * *$ & $5.41^{*}$ & $-5.77^{*}$ & $7.78^{* *}$ & $-5.93 * *$ & $6.50 * *$ & $20.58^{* * *}$ & $16.27 * *$ & $65.48^{* * *}$ & $57.06^{* * *}$ & $38.93 * *$ & $33.10^{* * *}$ \\
\hline $\mathbf{P}_{5} \times \mathbf{P}_{6}$ & $35.51^{* * *}$ & $41.48^{* * *}$ & $42.83^{* * *}$ & $50.24^{* * *}$ & $38.88^{* * *}$ & $45.50^{* * *}$ & $-24.12^{* * *}$ & $-22.62^{* *}$ & -1.10 & 1.69 & $-14.58^{* * *}$ & $-12.59 * *$ \\
\hline $\mathbf{P}_{5} \times \mathbf{P}_{7}$ & $-15.07^{* *}$ & $8.42 * *$ & -16.01 ** & $11.32^{\text {*** }}$ & -15.51 ** & $9.75^{* *}$ & $10.71^{* *}$ & $10.71^{* * *}$ & $49.15^{* * *}$ & $49.15^{* * *}$ & $26.57^{* * *}$ & $26.57 * *$ \\
\hline $\mathbf{P}_{5} \times \mathbf{P}_{8}$ & $20.22^{* * *}$ & $28.66 * *$ & $23.53^{* * *}$ & $33.73 * *$ & $21.75^{* * *}$ & $30.99 * *$ & $-15.22 * *$ & $-7.14^{*}$ & $11.94 * *$ & $27.12 * *$ & -3.77 & 6.99 \\
\hline $\mathbf{P}_{5} \times \mathbf{P}_{9}$ & $34.47 * *$ & $34.47 * *$ & $41.98 * *$ & $41.98 * *$ & $37.92^{* * *}$ & $37.92 * *$ & $-27.62 * *$ & $-17.86^{* * *}$ & $-9.00 *$ & 8.47 & $-19.72 * *$ & -6.99 \\
\hline $\mathbf{P}_{5} \times \mathbf{P}_{10}$ & $-6.45 * *$ & $4.61^{*}$ & $-6.21^{*}$ & $6.84 *$ & $-6.34 * *$ & $5.63 *$ & $17.86^{* * *}$ & $17.86^{* * *}$ & $59.32 * *$ & $59.32 * *$ & $34.97 * *$ & $34.97 * *$ \\
\hline $\mathbf{P}_{6} \times \mathbf{P}_{7}$ & $-16.01 * *$ & $7.21^{* * *}$ & $-17.08 * *$ & $9.91 * *$ & $-16.51^{* *}$ & $8.45 * *$ & $14.01 * *$ & $16.27^{* * *}$ & $52.75^{* * *}$ & $57.06^{* * *}$ & $30.07^{* * *}$ & $33.10 * *$ \\
\hline $\mathbf{P}_{6} \times \mathbf{P}_{8}$ & $23.03 * *$ & $31.66^{* *}$ & $28.10^{* * *}$ & 38.68 ** & $25.38^{* *}$ & $34.89 * *$ & $-18.48^{* * *}$ & $-10.71^{* *}$ & 4.48 & $18.64^{* * *}$ & $-8.81 * *$ & 1.40 \\
\hline $\mathbf{P}_{6} \times \mathbf{P}_{9}$ & $35.89^{* * *}$ & $41.88 * *$ & $15.02 * *$ & $20.99 * *$ & $26.27 * *$ & $32.29 * *$ & $-26.92 * *$ & $-17.06^{* * *}$ & $26.07 * *$ & $50.28 * *$ & -4.43 & $10.72 * *$ \\
\hline $\mathbf{P}_{6} \times \mathbf{P}_{10}$ & $21.51^{* * *}$ & $35.87 * *$ & $26.09^{* * *}$ & $43.63^{* * *}$ & $23.63^{* * *}$ & $39.44 * *$ & $-10.89 * *$ & -9.13 & $17.58^{* * *}$ & $20.90^{* *}$ & 0.91 & 3.26 \\
\hline $\mathbf{P}_{7} \times \mathbf{P}_{8}$ & $-7.22 * *$ & $18.44 * *$ & $-7.12 * *$ & $23.11^{* * *}$ & $-7.17 * *$ & $20.59 * *$ & -4.35 & 4.76 & $23.88^{* * *}$ & $40.68^{* * *}$ & $7.55^{*}$ & $19.58 * *$ \\
\hline $\mathbf{P}_{7} \times \mathbf{P}_{9}$ & $5.18 * *$ & $34.27 * *$ & $8.19^{* *}$ & $43.40 * *$ & $6.59 * *$ & $38.46 * *$ & $-27.27 * *$ & $-17.46 * *$ & 1.42 & $20.90 * *$ & $-15.09 * *$ & -1.63 \\
\hline $\mathbf{P}_{7} \times \mathbf{P}_{10}$ & -0.16 & $27.45^{* * *}$ & 1.96 & $35.14 * *$ & 0.83 & $30.99 * *$ & 3.45 & -4.76 & $39.49 * *$ & $23.73^{* * *}$ & $17.99^{* * *}$ & 6.99 \\
\hline$P_{8} \times P 9$ & $6.55^{* * *}$ & $14.03 * *$ & $8.93 * *$ & $17.92 * *$ & $7.65 * *$ & $15.82 * *$ & -0.35 & $13.10^{* * *}$ & $27.96 * *$ & $52.54 * *$ & $11.67^{* * *}$ & $29.37 * *$ \\
\hline $\mathbf{P}_{8} \times \mathbf{P}_{10}$ & $-4.84^{*}$ & $6.41^{* *}$ & -4.35 & 8.96 ** & $-4.61 *$ & $7.58^{* * *}$ & 5.80 & $15.87^{* * *}$ & 40.30 ** & $59.32^{* * *}$ & $20.34 * *$ & $33.80^{\text {*** }}$ \\
\hline $\mathbf{P}_{9} \times \mathbf{P}_{10}$ & $9.14 * *$ & $22.04 * *$ & $11.80^{* * *}$ & 27.36 ** & $10.37 * *$ & $24.49 * *$ & $-26.92 * *$ & $-17.06^{* * *}$ & $-8.06^{*}$ & $9.60 *$ & $-18.91 * *$ & -6.06 \\
\hline $\begin{array}{l}\text { No. of crosses with } \\
\text { significant }(+) \text { heterosis }\end{array}$ & 24 & 43 & 23 & 44 & 24 & 44 & 12 & 15 & 33 & 40 & 25 & 30 \\
\hline $\begin{array}{c}\text { No. of crosses with } \\
\text { significant (-) 'heterosis }\end{array}$ & 18 & 0 & 17 & 0 & 18 & o & 17 & 14 & 4 & 2 & 11 & 4 \\
\hline Range of heterosis & -26.40 to 35.89 & 4.01 to 49.70 & -28.69 to 44.13 & -1.42 to 56.37 & -23.26 to 40.20 & -0.65 to 54.39 & -39.93 to 27.16 & -36.11 to 22.62 & -24.35 to 87.80 & -17.51 to 61.02 & -28.20 to 48.60 & -28.44 to40.56 \\
\hline
\end{tabular}

*, ** Significant at 5 per cent and 1 per cent probability levels, respectively. 
Table.1 Cont.....

\begin{tabular}{|c|c|c|c|c|c|c|c|c|c|c|c|c|}
\hline \multirow{3}{*}{ Crosses } & \multicolumn{6}{|c|}{ Total sugar (\%) } & \multicolumn{6}{|c|}{ Dry matter content in fruit } \\
\hline & \multicolumn{2}{|c|}{$E_{1}$} & \multicolumn{2}{|c|}{$\mathbf{E}_{2}$} & \multicolumn{2}{|c|}{ Pooled } & \multicolumn{2}{|c|}{$E_{1}$} & \multicolumn{2}{|c|}{$\begin{array}{ll} & \mathbf{E}_{2} \\
\text { BP }\end{array}$} & \multicolumn{2}{|c|}{ Pooled } \\
\hline & BP & SV & BP & SV & BP & SV & BP & SV & BP & SV & BP & SV \\
\hline $\mathbf{P}_{1} \times \mathbf{P}_{2}$ & $-4.89^{* *}$ & $8.79^{* *}$ & $4.23^{*}$ & $22.96^{* *}$ & -0.77 & $15.09^{* * *}$ & $-23.12^{* *}$ & $-15.05^{* *}$ & -2.58 & $-12.58^{* * *}$ & -6.78 & $-13.74 * *$ \\
\hline $\mathbf{P}_{1} \times \mathbf{P}_{3}$ & $8.84 * *$ & $16.38^{* * *}$ & $20.98^{* *}$ & $31.45^{* *}$ & $14.29 * *$ & $23.08 * *$ & $17.03 * *$ & $11.20 * *$ & $12.64 * *$ & 1.08 & $14.76^{* * *}$ & 5.82 \\
\hline$\overline{P_{1} \times \mathbf{P}_{4}}$ & $7.47 * *$ & $14.91^{* * *}$ & $27.57^{* * *}$ & $38.60^{* * *}$ & $16.48^{* * *}$ & $25.44 * *$ & $-20.45^{* * *}$ & $-16.27 * *$ & $-15.93^{* * *}$ & $-12.04 * *$ & $-18.05^{* *}$ & $-14.02^{* * *}$ \\
\hline $\mathbf{P}_{1} \times \mathbf{P}_{5}$ & $8.48^{* *}$ & $12.38^{* * *}$ & $26.11^{* * *}$ & $31.78^{* * *}$ & $16.36^{* * *}$ & $21.01 * *$ & $13.91 * *$ & $13.91 * *$ & 1.47 & 1.47 & $7.30^{*}$ & $7.30^{*}$ \\
\hline $\mathbf{P}_{1} \times \mathbf{P}_{6}$ & $16.32^{* * *}$ & $20.51^{* *}$ & $30.73^{* * *}$ & $36.61^{* *}$ & $22.76^{* * *}$ & $27.66 * *$ & $-13.26^{* * *}$ & $-17.59 * *$ & -5.07 & $-14.81^{* *}$ & $-9.03^{*}$ & $-16.11 * *$ \\
\hline $\mathbf{P}_{1} \times \mathbf{P}_{7}$ & 1.32 & $12.65 * *$ & $12.41 * *$ & $28.12 * *$ & $6.32 * *$ & $19.53^{* *}$ & $-10.41 * *$ & $-14.87 * *$ & -4.90 & -1.23 & -5.81 & $-7.63^{*}$ \\
\hline $\mathbf{P}_{1} \times \mathbf{P}_{8}$ & $8.64^{* * *}$ & $17.18^{* * *}$ & $20.61^{* * *}$ & $32.45^{* *}$ & $14.01 * *$ & $23.96 * *$ & -11.19 ** & -4.90 & $-7.48^{*}$ & -1.70 & $-9.23 *$ & -3.20 \\
\hline $\mathbf{P}_{1} \times \mathbf{P}_{9}$ & $12.34 * *$ & $16.38^{* *}$ & $25.80^{* * *}$ & $31.45^{* *}$ & $18.35^{* *}$ & $23.08^{* *}$ & $28.27 * *$ & $21.87^{* * *}$ & $30.56^{* * *}$ & $19.98^{* *}$ & $31.08^{* *}$ & $20.87^{* * *}$ \\
\hline $\mathbf{P}_{1} \times \mathbf{P}_{10}$ & 2.28 & $7.59 * *$ & $15.31^{* * *}$ & $22.80^{* *}$ & $8.11 * *$ & $14.35 * *$ & 4.25 & $\begin{array}{l}0.79 \\
\end{array}$ & 7.15 & $\begin{array}{l}4.01 \\
\end{array}$ & 5.80 & 2.50 \\
\hline $\mathbf{P}_{2} \times \mathbf{P}_{3}$ & 2.33 & $17.04 * *$ & $12.13^{* * *}$ & $32.28^{* *}$ & $6.76^{* *}$ & $23.82^{* * *}$ & $-23.12^{* *}$ & $-15.05^{* * *}$ & $9.66^{*}$ & $-15.90^{* * *}$ & $-8.68^{*}$ & $-15.50^{* * *}$ \\
\hline $\mathbf{P}_{2} \times \mathbf{P}_{4}$ & -0.58 & $13.72^{* * *}$ & $8.60^{* *}$ & $28.12^{* * *}$ & $3.57^{*}$ & $20.12^{* * *}$ & 0.16 & $10.67^{* *}$ & $-24.26^{* * *}$ & $-20.76^{* * *}$ & $-10.43^{* * *}$ & $\begin{array}{l}-6.03 \\
\end{array}$ \\
\hline $\mathbf{P}_{2} \times \mathbf{P}_{5}$ & $-10.83^{* *}$ & 2.00 & -3.81 & $13.48^{* *}$ & $-7.65^{* *}$ & $7.10^{* *}$ & $-27.55^{* *}$ & $-19.95^{* *}$ & $-16.90^{* * *}$ & $-16.90^{* * *}$ & $-18.33^{* * *}$ & $-18.33^{* * *}$ \\
\hline $\mathbf{P}_{2} \times \mathbf{P}_{6}$ & 1.86 & $16.51^{* *}$ & $11.57^{* * *}$ & $31.61^{* * *}$ & $6.25 * *$ & $23.22 * *$ & $-18.05 * *$ & $-9.45^{* * *}$ & $18.05^{* * *}$ & $\begin{array}{l}-7.64 * \\
\end{array}$ & $\begin{array}{l}-1.11 \\
\end{array}$ & $-8.49 *$ \\
\hline $\mathbf{P}_{2} \times \mathbf{P}_{7}$ & 1.63 & $16.25 * *$ & $11.28^{* *}$ & $31.28 * *$ & $5.99 * *$ & $22.93 * *$ & $-29.93 * *$ & $-22.57 * *$ & $-22.21 * *$ & $-19.21^{* * *}$ & $-19.23^{* *}$ & $-20.79 * *$ \\
\hline $\mathbf{P}_{2} \times \mathbf{P}_{8}$ & 0.58 & $15.05^{* * *}$ & $10.01^{* *}$ & $29.78^{* * *}$ & $4.85^{* *}$ & 21.60 ** & $-15.12 * *$ & $-6.21^{*}$ & $-10.38 * *$ & -4.78 & $-11.34 * *$ & -5.45 \\
\hline $\mathbf{P}_{2} \times \mathbf{P}_{9}$ & 0.12 & $14.51^{* *}$ & $9.45^{* *}$ & $29.12^{* *}$ & $\begin{array}{l}4.34^{*} \\
\end{array}$ & $21.01^{* *}$ & $9.42 * *$ & $20.91 * *$ & $29.64 * *$ & $19.14 * *$ & $29.64 * *$ & $19.97^{* * *}$ \\
\hline $\mathbf{P}_{2} \times \mathbf{P}_{10}$ & 0.23 & $14.65 * *$ & $9.59 * *$ & $29.28^{* * *}$ & $4.46^{*}$ & $21.15^{* * *}$ & $-12.75^{* *}$ & -3.59 & 0.48 & -2.47 & 0.13 & -2.99 \\
\hline $\mathbf{P}_{3} \times \mathbf{P}_{4}$ & $9.84^{* * *}$ & $17.44 * *$ & $22.21^{* *}$ & $32.78 * *$ & $15.38^{* * *}$ & $24.26^{* * *}$ & 3.49 & $8.92 * *$ & 3.76 & $8.56^{*}$ & 3.63 & $8.73 * *$ \\
\hline $\mathbf{P}_{3} \times \mathbf{P}_{5}$ & $12.70^{* * *}$ & $20.51^{* *}$ & $25.73^{* *}$ & $36.61 * *$ & $18.54 * *$ & $27.66 * *$ & $-12.60^{* *}$ & $-12.60^{* *}$ & $-10.42^{* * *}$ & $-10.42^{* *}$ & $-11.44 * *$ & $-11.44 * *$ \\
\hline $\mathbf{P}_{3} \times \mathbf{P}_{6}$ & 3.24 & $10.39^{* * *}$ & $14.09^{* * *}$ & $23.96^{* * *}$ & $8.10^{* *}$ & $16.42^{* * *}$ & $53.77^{* * *}$ & $15.84^{* *}$ & $46.55^{* * *}$ & $14.66^{* * *}$ & $49.87^{* * *}$ & $15.21^{* * *}$ \\
\hline $\mathbf{P}_{3} \times \mathbf{P}_{7}$ & $8.74 * *$ & $20.91 * *$ & $20.29 * *$ & $37.10^{* *}$ & $13.95^{* * *}$ & $28.11^{* *}$ & $-40.54 * *$ & $-45.58 * *$ & $-18.05 * *$ & $-14.89 * *$ & $-27.88^{* * *}$ & $-29.27 * *$ \\
\hline $\mathbf{P}_{3} \times \mathbf{P}_{8}$ & $7.78 * *$ & $16.25^{* * *}$ & $19.55^{* * *}$ & $31.28^{* * *}$ & $13.06^{* * *}$ & $22.93 * *$ & $-18.22^{* * *}$ & $-12.42^{* *}$ & $-18.01 * *$ & $-12.89^{* *}$ & $-18.11^{* * *}$ & $-12.67 * *$ \\
\hline $\mathbf{P}_{3} \times \mathbf{P}_{9}$ & $6.35^{* * *}$ & $13.72 * *$ & $16.69 * *$ & $26.79 * *$ & $10.99 * *$ & $19.53^{* *}$ & 0.87 & $-8.40^{* * *}$ & -4.70 & $-12.42 * *$ & -2.11 & $-10.54 * *$ \\
\hline $\mathbf{P}_{3} \times \mathbf{P}_{10}$ & $5.23 * *$ & $12.52^{* * *}$ & $16.54^{* * *}$ & $26.62^{* * *}$ & $10.30^{* * *}$ & $18.79 * *$ & $-8.69^{* * *}$ & $-11.72^{* *}$ & $\begin{array}{l}-7.31 \\
\end{array}$ & $-10.03^{* * *}$ & $-7.96^{* * *}$ & $-10.82^{* * *}$ \\
\hline $\mathbf{P}_{4} \times \mathbf{P}_{5}$ & $9.09^{* *}$ & $16.64 * *$ & $16.39^{* * *}$ & $26.46^{* *}$ & $12.36^{* * *}$ & $21.01^{* *}$ & $-21.70^{* *}$ & $-17.59^{* *}$ & $8.92^{*}$ & $13.97^{* * *}$ & -5.47 & -0.82 \\
\hline $\mathbf{P}_{4} \times \mathbf{P}_{6}$ & $12.83^{* * *}$ & $20.64 * *$ & 21.59 *** & $32.11^{* *}$ & $16.76^{* * *}$ & $25.74 * *$ & $-8.89 * *$ & -4.11 & 2.95 & $7.72^{*}$ & -2.62 & 2.17 \\
\hline $\mathbf{P}_{4} \times \mathbf{P}_{7}$ & -0.84 & $10.25^{* *}$ & $8.61 * *$ & $23.79^{* * *}$ & 3.42 & $16.27^{* * *}$ & $-17.71 * *$ & $-13.39 * *$ & $-25.96^{* * *}$ & $-22.53^{* *}$ & $-22.08 * *$ & $-18.25^{* * *}$ \\
\hline $\mathbf{P}_{4} \times \mathbf{P}_{8}$ & $7.78 * *$ & $16.25 * *$ & $19.55^{* * *}$ & $31.28^{* * *}$ & $13.06^{* * *}$ & $22.93 * *$ & $6.37^{*}$ & $13.91 * *$ & 6.32 & $12.96^{* * *}$ & $\begin{array}{l}6.34 * * \\
\end{array}$ & $13.41^{* * *}$ \\
\hline $\mathbf{P}_{4} \times \mathbf{P}_{9}$ & $10.09^{* * *}$ & $17.71^{* * *}$ & $22.51^{* * *}$ & $33.11 * *$ & $15.66 * *$ & $24.56^{* * *}$ & 0.83 & $6.12 *$ & $-15.27 * *$ & $-11.34 * *$ & $-7.70 * *$ & -3.16 \\
\hline$\overline{\mathbf{P}_{4} \times \mathbf{P}_{10}}$ & 1.99 & $9.05^{* *}$ & $12.56^{* * *}$ & $22.30^{* * *}$ & $6.73 * *$ & $14.94 * *$ & $-5.74 *$ & $\begin{array}{l}-0.79 \\
\end{array}$ & $-28.47 * *$ & $-25.15^{* * *}$ & $-17.78^{\text {*** }}$ & $-13.74 * *$ \\
\hline $\mathbf{P}_{5} \times \mathbf{P}_{6}$ & $15.81^{* * *}$ & $19.97 * *$ & $30.10^{* * *}$ & $35.94^{* * *}$ & $22.19^{* * *}$ & $27.07 * *$ & $-23.71 * *$ & $-23.71^{* * *}$ & $-8.56^{*}$ & $-8.56^{*}$ & $-15.66^{* * *}$ & $-15.66^{* * *}$ \\
\hline $\mathbf{P}_{5} \times \mathbf{P}_{7}$ & $\begin{array}{l}-1.80 \\
\end{array}$ & $9.19 * *$ & $7.45^{* *}$ & $22.46 * *$ & 2.37 & $15.09 * *$ & 6.91* & $6.91 *$ & 2.82 & 6.79 & 6.85 & 6.85 \\
\hline $\mathbf{P}_{5} \times \mathbf{P}_{8}$ & $8.15^{* *}$ & $16.64^{* * *}$ & $20.00^{* * *}$ & $31.78^{* *}$ & $13.47^{* * *}$ & $23.37^{* * *}$ & $-11.93^{* *}$ & -5.69 & $-20.55^{* * *}$ & $-15.59^{* * *}$ & $-16.49^{* * *}$ & $-10.95^{* * *}$ \\
\hline $\mathbf{P}_{5} \times \mathbf{P}_{9}$ & $15.83^{* * *}$ & $16.91 * *$ & $30.59^{* *}$ & $32.11^{* *}$ & $22.40^{* * *}$ & $23.67 * *$ & -0.17 & -0.17 & $-18.75^{* * *}$ & $-18.75^{* * *}$ & $-10.05^{* *}$ & $-10.05 * *$ \\
\hline $\mathbf{P}_{5} \times \mathbf{P}_{10}$ & $3.67^{*}$ & $9.05^{* *}$ & $14.84^{* * *}$ & $22.30^{* * *}$ & $8.67^{* *}$ & $14.94 * *$ & $-14.70^{* * *}$ & $-14.70^{* * *}$ & $-9.95 * *$ & $-9.95 * *$ & $-12.18^{* * *}$ & $-12.18^{* * *}$ \\
\hline $\mathbf{P}_{6} \times \mathbf{P}_{7}$ & -0.84 & $10.25 * *$ & $8.61 * *$ & $23.79 * *$ & 3.42 & $16.27 * *$ & -3.54 & $-11.72^{* * *}$ & $9.36 * *$ & $13.58^{* * *}$ & 3.72 & 1.72 \\
\hline $\mathbf{P}_{6} \times \mathbf{P}_{8}$ & $8.89^{* * *}$ & $17.44 * *$ & $20.91^{* *}$ & $32.78^{* *}$ & 14.29 ** & $24.26 * *$ & $-11.76 * *$ & -5.51 & -0.15 & 6.10 & -5.61 & 0.66 \\
\hline $\mathbf{P}_{6} \times \mathbf{P}_{9}$ & $17.87^{* * *}$ & $22.10^{* * *}$ & 24.04 ** & $29.62^{* * *}$ & $20.63^{* * *}$ & $25.44 * *$ & -5.78 & $-14.44 * *$ & -3.02 & -10.88 ** & -4.31 & $-12.55^{* * *}$ \\
\hline $\mathbf{P}_{6} \times \mathbf{P}_{10}$ & $14.81^{* * *}$ & $20.77 * *$ & $28.59^{* * *}$ & $36.94 * *$ & $20.98^{* * *}$ & $27.96 * *$ & 4.89 & 1.40 & 5.01 & 1.93 & 4.95 & 1.68 \\
\hline$\overline{\mathbf{P}_{7} \times \mathbf{P}_{8}}$ & 2.40 & $13.85 * *$ & $12.55^{* * *}$ & $28.29 * *$ & $6.97 * *$ & $20.27 * *$ & $-17.57 * *$ & $-11.72 * *$ & $-14.96^{* * *}$ & $-9.65 * *$ & $-16.19 * *$ & $-10.62 * *$ \\
\hline $\mathbf{P}_{7} \times \mathbf{P}_{9}$ & $5.15^{* * *}$ & $16.91^{* *}$ & $20.00^{* * *}$ & $36.77^{* *}$ & $11.84^{* * *}$ & $25.74 * *$ & $17.97^{* * *}$ & $7.96 * *$ & $-21.77 * *$ & $-18.75^{* * *}$ & -4.39 & -6.23 \\
\hline $\mathbf{P}_{7} \times \mathbf{P}_{10}$ & $4.91^{* *}$ & $16.64 * *$ & $15.62 * *$ & $31.78^{* * *}$ & $9.74 * *$ & $23.37 * *$ & -0.45 & -3.76 & $-7.88 *$ & -4.32 & -2.17 & -4.06 \\
\hline$\overline{P_{8} \times \mathbf{P 9}}$ & $5.43 * *$ & $13.72^{* * *}$ & $16.67^{* * *}$ & $28.12^{* * *}$ & $10.48^{* * *}$ & $20.12 * *$ & $-6.94 *$ & -0.35 & $-24.26^{* * *}$ & $-19.52^{* * *}$ & $-16.11^{\text {*** }}$ & $-10.54 * *$ \\
\hline $\mathbf{P}_{8} \times \mathbf{P}_{10}$ & 1.60 & $9.59 * *$ & $12.73^{* * *}$ & $23.79^{* *}$ & $6.60^{* * *}$ & $15.90^{* *}$ & $-5.64 *$ & 1.05 & $20.84 * *$ & $28.40 * *$ & $8.38 * *$ & $15.58^{* * *}$ \\
\hline $\mathbf{P}_{9} \times \mathbf{P}_{10}$ & $3.54 *$ & $8.92^{* *}$ & $14.69^{* * *}$ & $22.13^{* * *}$ & $8.53 * *$ & $14.79^{* *}$ & 1.45 & -1.92 & $8.43^{*}$ & 5.25 & 5.16 & 1.89 \\
\hline $\begin{array}{c}\text { No. of crosses with } \\
\text { significant }(+) \text { heterosis }\end{array}$ & 27 & 43 & 44 & 45 & 40 & 45 & 8 & 11 & 10 & 9 & 7 & 7 \\
\hline $\begin{array}{c}\text { No. of crosses with } \\
\text { significant (-) 'heterosis }\end{array}$ & 2 & o & o & o & 1 & o & 26 & 21 & 20 & 24 & 21 & 21 \\
\hline Range of heterosis & -10.83 to 17.87 & 2.00 to 22.10 & -3.81 to 30.73 & \begin{tabular}{|l|l|}
13.48 to 38.60 \\
\end{tabular} & -7.65 to 22.76 & 7.10 to 28.11 & \begin{tabular}{|l|l|}
-40.54 to 53.77 \\
\end{tabular} & -23.71 to 21.87 & -28.47 to 46.55 & -25.15 to 28.40 & -27.88 to 49.87 & -29.27 to 20.87 \\
\hline
\end{tabular}

*, ** Significant at 5 per cent and 1 per cent probability levels, respectively. 
Thirty five crosses over better parent and forty crosses over standard parents showed significant heterosis over seasons for fruit yield (Tables 2 and 3). Increased yield in crosses of bottle gourd observed in present investigation is in conformity with the findings of various workers (Janakiram and Sirohi, 1992, Singh et al., 1996 Sirohi et al., 2005 and Gayakawad et al., (2016)). Fruit yield per plant being complex trait is a multiplicative product of several basic component traits of yield. The improvement in heterosis for yield component may not necessarily be reflected in increased yield. Contrarily the increased fruit yield will definitely be cause of increase in one or more component traits. In the present study, the best performing heterobeltiotic $\mathrm{F}_{1}\left(\mathrm{P}_{3} \times \mathrm{P}_{9}\right)$ for yield common over seasons also showed significant and top ranked heterobeltiosis for number of fruits per plant in over seasons. This hybrid also showed significant and desirable heterosis for fruit circumference and fruit weight. Likewise, out of twenty seven crosses found significant heterotic over standard parent common over seasons. Among top heterotic crosses some of the parents were more frequently involved. The above findings indicated that some inbreds had more heterotic capability compared to other ones during hybridization process. As the performance of hybrids depends upon the heterotic capability of parents involved, from economic point of view it will be useful to select and utilize the parental inbreds with strong heterotic capability for important economic traits associated with yield in order to achieve higher gains in $F_{1}$ hybrids through exploitation of heterosis.

A perusal of table 1 which showed best five crosses on the basis of desirable significant heterobeltiosis, per se performance and common crosses for eighteen traits in pooled revealed that common crosses on the basis of per se performance and better parent heterosis for fruit yield per plant were $\mathrm{P}_{3} \times \mathrm{P}_{9}, \mathrm{P}_{5} \times \mathrm{P}_{8}$, $\mathrm{P}_{4} \times \mathrm{P}_{5}, \mathrm{P}_{8} \times \mathrm{P}_{10}$ and $\mathrm{P}_{7} \times \mathrm{P}_{9}$ were common crosses on the basis of per se performance and out of these commen crosses $\mathrm{P}_{6} \times \mathrm{P}_{9}, \mathrm{P}_{5} \times \mathrm{P}_{8}$ and $\mathrm{P}_{4} \times \mathrm{P}_{5}$ were also found common in respect to per se performance and better parent heterosis for fruit yield as well as some other traits studied. Standard heterosis of five best crosses combinations along with per se performance and common crosses for different characters had been presented in table 1 the extent of heterosis of five best crosses (56.80 to $69.17 \%$ ) fruit yield per plant revealed that there was a great scope of realizing higher yield in bottle gourd through heterosis breeding.

\section{Acknowledgment}

The work on bottle gourd reported in this paper has been supported by research and teaching faculties of Department of Vegetable Science, N.D.U.A.T and We would also like to thank Mr. Murli Mohan Khetan for statistical analysis.

\section{References}

Cutler, H. C. and Whitaker, T. W. (1961). History and distribution of the cultivated cucurbits in the Americas. American Antiquity, 26: 469-485.

Dubey, S.K. and Maurya, I.B. (2003). Studies on heterosis and combining ability in bottle gourd (Lagenaria siceraria (Molina) Standl.). Indian J. Genet., 63: 148-152

Gayakawad, P. S., Evoor, S., Mulge, R., Reshmika, P. K. and Nagesh, G. C. (2016).Heterosis studies in bottle gourd [Lagenaria siceraria (Mol.) Standl.] for growth and yield parameters. Environment and Ecology; 2016. 34(4):1756-1763. 11

Ghuge, M. B., Syamal, M. M. and Karcho, S. (2016). Heterosis in bottle gourd 
[Lagenaria siceraria (Mol.) Standl.]. Indian Journal of Agricultural Research; 50(5):466-470. 5

Hayes, H.K., Immer, F. and Smith, D.C. (1955). Methods of Plant Breeding. Mc Graw-Hill Book Co., Inc. New York. pp. 52-66.

Janakiram, T. and Sirohi, P.S. (1992). Studies on heterosis for quantitative characters in bottle gourd. J. Maharashtra Agric. Univ., 17: 204-206.

Kumar, S., Singh, S.P. and Singh, N.K. (1998). Line $\times$ tester analysis for combining ability and heterosis in bottle gourd. Veg. Sci., 25: 78-80.

Maurya, P.K. (2010). Heterosis and combining ability analysis in bottle gourd [Lagenaria siceraria (Molina) Standl.] M.Sc. Thesis, Submitted to N.D. Uni. of Agric. \& Tech. Kumarganj, Faizabad.

Sirohi, P.S., Sivakami, N. and Choudhary, B. (1985). Heterosis in long-fruited bottle gourd. Ann. Agric. Res., 6: 210-214.
Sirohi, S., Rana, S.C. and Rajkumar. (2005). Heterosis study in bottle gourd (Lagenaria siceraria (Mol.) Standl.). In: National Seminar on cucurbits, Sept. 22-23, G.B. Pant University of Agriculture and Technology, Pantnagar. p. 39.

Singh, R.K. (2008). Studies on heterosis, combining ability and gene action in bottle gourd [Lagenaria siceraria (Molina) Standl.] M.Sc. Thesis, Submitted to N.D. Uni. of Agric. \& Tech. Kumarganj, Faizabad.

Singh, S.P., Maurya, I.B. and Singh, N.K. (1996). Occurrence of andromonoecious form in bottle gourd exhibiting monogenic recessive inheritance. Curr. Sci., 70: 458-459.

Thamburaj, S. and Singh, N. (2005). Vegetables, Tubercrops and Spices, Directorate of Information and Publications of Agriculture, ICAR, New Delhi. pp. 271-272.

\section{How to cite this article:}

Deepak Kumar Gautam, G.C. Yadav, Pushpendra Kumar, Vimlesh Kumar and Manvendra Singh. 2017. Estimation of Heterosis for Growth, Yield and Quality Traits in Bottle Gourd [Lagenaria Siceraria (Mol.) Standl.]. Int.J.Curr.Microbiol.App.Sci. 6(8): 789-802. doi: https://doi.org/10.20546/ijcmas.2017.608.100 